\title{
A Globally Convergent Inexact Newton-Like Cayley Transform Method for Inverse Eigenvalue Problems
}

\author{
Yonghui Ling ${ }^{1}$ and Xiubin $\mathrm{Xu}^{2}$ \\ ${ }^{1}$ Department of Mathematics, Zhejiang University, Hangzhou 310027, China \\ ${ }^{2}$ Department of Mathematics, Zhejiang Normal University, Jinhua 321004, China \\ Correspondence should be addressed to Yonghui Ling; yhling@zju.edu.cn
}

Received 22 July 2013; Accepted 28 October 2013

Academic Editor: Nicola Mastronardi

Copyright (c) 2013 Y. Ling and X. Xu. This is an open access article distributed under the Creative Commons Attribution License, which permits unrestricted use, distribution, and reproduction in any medium, provided the original work is properly cited.

We propose an inexact Newton method for solving inverse eigenvalue problems (IEP). This method is globalized by employing the classical backtracking techniques. A global convergence analysis of this method is provided and the R-order convergence property is proved under some mild assumptions. Numerical examples demonstrate that the proposed method is very effective in solving the IEP with distinct eigenvalues.

\section{Introduction}

In the present paper, we consider inverse eigenvalue problems (IEP) which are defined as follows. Let $\mathbf{c}=\left(c_{1}, c_{2}, \ldots, c_{n}\right)^{\top} \in$ $\mathbb{R}^{n}$ and let $\left\{A_{i}\right\}_{i=0}^{n}$ be a sequence of real symmetric $n \times$ $n$ matrices. Define

$$
A(\mathbf{c})=A_{0}+\sum_{i=1}^{n} c_{i} A_{i}
$$

and denote its eigenvalues by $\left\{\lambda_{i}(\mathbf{c})\right\}_{i=1}^{n}$ with the increasing order $\lambda_{1}(\mathbf{c}) \leq \lambda_{2}(\mathbf{c}) \leq \cdots \leq \lambda_{n}(\mathbf{c})$. Given $n$ real numbers $\left\{\lambda_{i}^{*}\right\}_{i=1}^{n}$ which are arranged in increasing order $\lambda_{1}^{*} \leq$ $\lambda_{2}^{*} \leq \cdots \leq \lambda_{n}^{*}$, the IEP is to find a vector $\mathbf{c}^{*} \in \mathbb{R}^{n}$ such that

$$
\lambda_{i}\left(\mathrm{c}^{*}\right)=\lambda_{i}^{*}, \quad i=1,2, \ldots, n .
$$

Such vector $\mathrm{c}^{*}$ is called a solution of the IEP. This type of inverse problem arises in a variety of applications, for instance, the inverse Toeplitz eigenvalue problem $[1,2]$, inverse Sturm-Liouville's problem, inverse vibrating string problem, and the pole assignment problem; see [3-5] and the references therein for more details on these applications.

Define $f: \mathbb{R}^{n} \rightarrow \mathbb{R}^{n}$ by

$$
f(\mathbf{c})=\left(\lambda_{1}(\mathbf{c})-\lambda_{1}^{*}, \lambda_{2}(\mathbf{c})-\lambda_{2}^{*}, \ldots, \lambda_{n}(\mathbf{c})-\lambda_{n}^{*}\right)^{\top} .
$$

Then, solving IEP (2) is equivalent to solving the nonlinear equation $f(\mathbf{c})=0$ on $\mathbb{R}^{n}$. It is clear that $\mathbf{c}^{*}$ is a solution of the IEP if and only if $\mathbf{c}^{*}$ is a solution of the equation $f(\mathbf{c})=0$. Based on this equivalence, Newton's method can be applied to the IEP, and it converges quadratically [6]. As it is known, each iteration of Newton's method involves solving a complete eigenproblem for the matrix $A(\mathbf{c})$. To overcome this drawback, different Newton-like methods have been proposed and studied $[7,8]$. To alleviate the over-solving problem, Bai et al. presented in [9] an inexact Cayley transform method for solving the nonlinear system $f(\mathbf{c})=$ 0 . To avoid the computation of the approximate Jacobian equations, Shen and Li proposed in $[10,11] \mathrm{Ulm}$-like methods for solving the IEPs. However, all these numerical methods for solving the IEP converge only locally.

In this paper, we study the numerical methods with global convergence property for solving the IEP. Since the IEP is a nonlinear equation, we review some classical work on solving the general nonlinear equation $f(\mathbf{c})=0$. Among the inexact Newton-type methods where a line search procedure is used, an inexact Newton backtracking method was proposed in [12]. It performed backtracking along the inexact Newton step, and computational results on a large set of test problems have shown its robustness and efficiency $[13,14]$.

The purpose of the present paper is motivated by the inexact Newton backtracking method proposed in [12], 
to propose an inexact Newton-type method which combines with the Cayley transform method for solving the IEP. In the backtracking procedure, we use the Rayleigh quotient instead of the classical merit function and therefore reduce the computational cost. Under the classical assumption, which is also used in $[7,9,10]$, that the given eigenvalues are distinct and the Jacobian matrix $J\left(\mathbf{c}^{*}\right)$ is invertible, we show that this method is globally convergent. Some numerical examples are reported to illustrate the effectiveness of the proposed method with distinct eigenvalues.

The paper is organized as follows. In Section 2, a global inexact Newton-type algorithm is proposed. The global convergence analysis is given in Section 3. And finally in Section 4, some numerical examples are given to confirm the numerical effectiveness and the good performance of our algorithm.

\section{A Globally Inexact Newton-Like Cayley Transform Method}

In this section, we present our algorithm. Let $\mathbb{R}^{n \times n}$ denote the set of all real $n \times n$ matrices. Let $\|\cdot\|$ and $\|\cdot\|_{F}$ denote the 2 -norm and the Frobenius norm in $\mathbb{R}^{n}$, respectively. The induced 2-norm in $\mathbb{R}^{n \times n}$ is also denoted by $\|\cdot\|$; that is,

$$
\|A\|:=\sup _{\mathbf{x} \in \mathbb{R}^{n}, \mathbf{x} \neq 0} \frac{\|A \mathbf{x}\|}{\|\mathbf{x}\|}, \quad A \in \mathbb{R}^{n \times n} .
$$

Then, we have $\|A\| \leq\|A\|_{F}$ for any $A \in \mathbb{R}^{n \times n}$. For $\mathbf{x} \in$ $\mathbb{R}^{n}$ and a positive number $r$, we use $\mathbf{B}(\mathbf{x}, r)$ to stand for the open ball with radius $r$ and center $\mathbf{x}$. Let $\lambda_{1}(\mathbf{c}) \leq \lambda_{2}(\mathbf{c}) \leq$ $\cdots \leq \lambda_{n}(\mathbf{c})$ be the eigenvalues of matrix $A(\mathbf{c})$ and let $\left\{\mathbf{q}_{i}(\mathbf{c})\right\}_{i=1}^{n}$ be the normalized eigenvectors corresponding to $\left\{\lambda_{i}(\mathbf{c})\right\}_{i=1}^{n}$. Define $J(\mathbf{c})=\left([J(\mathbf{c})]_{i j}\right)$ by

$$
[J(\mathbf{c})]_{i j}:=\mathbf{q}_{i}(\mathbf{c})^{\top} A_{j} \mathbf{q}_{i}(\mathbf{c}), \quad 1 \leq i, j \leq n .
$$

Let $\left\{\lambda_{i}^{*}\right\}_{i=1}^{n}$ be given with $\lambda_{1}^{*} \leq \lambda_{2}^{*} \leq \cdots \leq \lambda_{n}^{*}$ and write $\lambda^{*}=$ $\left(\lambda_{1}^{*}, \lambda_{2}^{*}, \ldots, \lambda_{n}^{*}\right)^{\top}$.

The Cayley transform method for computing approximately the eigenproblem of the matrix $A(\mathbf{c})$ was proposed in $[6]$ and was applied in $[9,10]$. We now recall this method and then apply it to our algorithm. Suppose that $c^{*}$ is a solution of the IEP. Then, there exists an orthogonal matrix $Q_{*}$ such that

$$
Q_{*}^{\top} A\left(\mathbf{c}^{*}\right) Q_{*}=\operatorname{diag}\left\{\lambda_{1}^{*}, \lambda_{2}^{*}, \ldots, \lambda_{n}^{*}\right\} \triangleq \Lambda^{*} .
$$

Assume that $\mathbf{c}, \boldsymbol{\rho}$, and $P$ are the current approximations of $\mathrm{c}^{*}, \lambda^{*}$, and $Q_{*}$, respectively. Define $e^{Y}:=P^{\top} Q_{*}$, where $Y$ is a skew-symmetric matrix. Then, (6) can be rewritten as

$$
\begin{aligned}
P^{\top} A\left(\mathbf{c}^{*}\right) P & =e^{Y} \Lambda^{*} e^{-Y} \\
& =\left(\mathbf{I}+Y+\frac{1}{2} Y^{2}+\cdots\right) \Lambda^{*}\left(\mathbf{I}-Y+\frac{1}{2} Y^{2}+\cdots\right) \\
& =\Lambda^{*}+Y \Lambda^{*}-\Lambda^{*} Y+O\left(\|Y\|^{2}\right) .
\end{aligned}
$$

Based on (7), we define the new approximation $c^{\text {new }}$ of $c^{*}$ by neglecting the second-order terms in $Y$ :

$$
P^{\top} A\left(\mathbf{c}^{\text {new }}\right) P=\Lambda^{*}+Y \Lambda^{*}-\Lambda^{*} Y .
$$

By equating the diagonal elements in (8), we have

$$
\lambda_{i}^{*}=\mathbf{p}_{i}(\mathbf{c})^{\top} A\left(\mathbf{c}^{\text {new }}\right) \mathbf{p}_{i}(\mathbf{c}), \quad i=1,2, \ldots, n,
$$

where $\left\{\mathbf{p}_{i}(\mathbf{c})\right\}_{i=1}^{n}$ are the column vectors of $P$. Thus, once we get $c^{\text {new }}$ by solving the Jacobian equation, we can obtain $Y$ by equating the off-diagonal elements in (8); that is,

$$
[Y]_{i j}:=\frac{\mathbf{p}_{i}(\mathbf{c})^{\top} A\left(\mathbf{c}^{\text {new }}\right) \mathbf{p}_{j}(\mathbf{c})}{\lambda_{j}^{*}-\lambda_{i}^{*}}, \quad 1 \leq i \neq j \leq n .
$$

In order to update the new approximation $P^{\text {new }}$ of $Q_{*}$, we construct an orthogonal matrix $U$ using Cayley's transform

$$
U:=\left(\mathbf{I}+\frac{1}{2} Y\right)\left(\mathbf{I}-\frac{1}{2} Y\right)^{-1}
$$

and set $P^{\text {new }}=P U$; that is, we can obtain $P^{\text {new }}$ by solving

$$
\left(\mathbf{I}+\frac{1}{2} Y\right) P^{\text {new }}=\left(\mathbf{I}-\frac{1}{2} Y\right) P .
$$

Finally, the new approximations of eigenvalues can be obtained by

$$
\rho_{i}^{\text {new }}:=\mathbf{p}_{i}\left(\mathbf{c}^{\text {new }}\right)^{\top} A\left(\mathbf{c}^{\text {new }}\right) \mathbf{p}_{i}\left(\mathbf{c}^{\text {new }}\right), \quad i=1,2, \ldots, n,
$$

where $\left\{\mathbf{p}_{i}\left(\mathbf{c}^{\text {new }}\right)\right\}_{i=1}^{n}$ are the column vectors of $P^{\text {new }}$.

Note that (12) can be computed as follows. Compute $H$ := $(I-(1 / 2) Y) P^{\top}$ and let $\mathbf{h}_{i}$ be the $i$ th column of $H$ at first. Then, solve $\mathbf{w}_{i}, i=1,2, \ldots, n$, iteratively from the $n$ linear systems:

$$
\left(I+\frac{1}{2} Y\right) \mathbf{w}_{i}=\mathbf{h}_{i}, \quad i=1,2, \ldots, n .
$$

Finally, set $P^{\text {new }}:=\left[\mathbf{w}_{1}, \ldots, \mathbf{w}_{n}\right]^{\top}$. Since $P$ is an orthogonal matrix and $Y$ is skew-symmetric matrix, we see that $P^{\text {new }}$ must be orthogonal. To maintain the orthogonality of $P^{\text {new }}$, (14) cannot be solved inexactly. One could expect that it requires only few iterations to solve each system of (14). This is due to the fact that as $\left\{\mathbf{c}^{k}\right\}$ converges to $\mathbf{c}^{*},\left\|Y_{k}\right\|$ converges to zero; see [6, (3.64)]. Consequently, the coefficient matrix on the left-hand side of (14) approaches the identity matrix in the limit.

For solving the general nonlinear equation $f(\mathbf{x})=0$, linesearch techniques [15] are often used to enlarge the convergence basin of a locally convergent method. They are based on a globally convergent method for a problem of the form $\min _{\mathbf{x} \in \mathbb{R}^{n}} M(\mathbf{x})$, where $M$ is an appropriately chosen merit function whose global minimum is a zero of $f$. In these cases, for a given direction $\mathbf{s} \in \mathbb{R}^{n}$, we have the iteration form $\mathbf{x}_{k+1}=\mathbf{x}_{k}+\alpha \mathbf{s}$, where $\alpha \in(0,1]$ is such that $M\left(\mathbf{x}_{k}+\alpha \mathbf{s}\right)<$ $M\left(\mathbf{x}_{k}\right)$. The existence of such an $\alpha$ is ensured if there exists an $\alpha_{0}>0$ such that $M\left(\mathbf{x}_{k}+\alpha \mathbf{s}\right)<M\left(\mathbf{x}_{k}\right)$ for all $\alpha<\alpha_{0}$. 
In typical linesearch strategies, the step length $\alpha$ is chosen by using so-called backtracking approach. Among the backtracking method, inexact Newton backtracking methods (INB) [12] is a globally convergent process where the $k$ th iteration of an inexact Newton method is embedded in a backtracking strategy. The merit function $M$ of INB usually used $M:=\|f\|$; see, for example, $[12-14,16]$. Thanks to (3), this will involve computing $\lambda_{i}\left(\mathbf{c}^{k}\right)$ of $A\left(\mathbf{c}^{k}\right)$ which are costly to compute. Our intention here is to replace them by the Rayleigh quotient (see (13)). In Section 3 we will show that this replacement retain superlinear and global convergence.

The details of our algorithm for solving the IEP are specified as Algorithm 1. In Step 7, the following sufficient decrease in the merit function $\left\|\rho(\mathbf{c})-\lambda^{*}\right\|$ based on the Rayleigh quotient is provided:

$$
\left\|\boldsymbol{\rho}\left(\mathbf{c}^{k}+\Delta \mathbf{c}^{k}\right)-\lambda^{*}\right\| \leq\left(1-\xi\left(1-\eta_{k}\right)\right)\left\|\boldsymbol{\rho}\left(\mathbf{c}^{k}\right)-\lambda^{*}\right\| .
$$

The while loop in Step 7 is also called backtracking loop below. Note that if we set

$$
\bar{\eta}_{k}^{1}:=\frac{\left\|\rho\left(\mathrm{c}^{k}\right)-\lambda^{*}\right\|}{\left\|\lambda^{*}\right\|}, \quad \bar{\eta}_{k}^{2}:=\frac{\left\|\rho\left(\mathrm{c}^{k}\right)-\lambda^{*}\right\|}{\left\|\boldsymbol{\rho}\left(\mathrm{c}^{k-1}\right)-\lambda^{*}\right\|},
$$

when $\mathbf{c}^{k}$ is away from an accumulation point $\mathbf{c}^{*}$, then $\bar{\eta}_{k}^{1}>1$ or $\bar{\eta}_{k}^{2}>1$ may be possible. Accordingly, the forcing term $\bar{\eta}_{k}$ given in (20) guarantees that $\bar{\eta}_{k} \in[0,1)$ for each $k$.

\section{Convergence Analysis}

In this section, we analyze the global behavior of Algorithm 1. We will show that if the given eigenvalues are distinct and if there exists an accumulation point $\mathbf{c}^{*}$ of $\left\{\mathbf{c}^{k}\right\}$ such that the Jacobian matrix $J\left(\mathbf{c}^{*}\right)$ is invertible, then the iterations are guaranteed to remain near $\mathbf{c}^{*}$ and $\rho\left(\mathbf{c}^{*}\right)-\lambda^{*}=0, \mathbf{c}^{k} \rightarrow \mathbf{c}^{*}$ as $k \rightarrow \infty$. Furthermore, for $k$ sufficiently large, we have the equality $\boldsymbol{c}^{k+1}=\mathbf{c}^{k}+\Delta \overline{\mathbf{c}}^{k}$. Thus, we obtain that the ultimate rate of convergence is $\beta$ which depends on the choices of the $\bar{\eta}_{k}$ given in (20).

Algorithm 1 (Inexact Newton-Like Backtracking Cayley Transform Method for IEP). For any $\mathbf{c}^{0} \in \mathbb{R}^{n}, \eta_{\max } \in[0,1)$, $\xi \in(0,1), 0<\theta_{\min }<\theta_{\max }<1$. Compute the orthonormal eigenvectors $\left\{\mathbf{q}_{i}\left(\mathbf{c}^{0}\right)\right\}_{i=1}^{n}$ and the eigenvalues $\left\{\lambda_{i}\left(\mathbf{c}^{0}\right)\right\}_{i=1}^{n}$ of $A\left(\mathbf{c}^{0}\right)$. Let

$$
\begin{aligned}
& P_{0}:= {\left[\mathbf{p}_{1}\left(\mathbf{c}^{0}\right), \mathbf{p}_{2}\left(\mathbf{c}^{0}\right), \ldots, \mathbf{p}_{n}\left(\mathbf{c}^{0}\right)\right] } \\
&= {\left[\mathbf{q}_{1}\left(\mathbf{c}^{0}\right), \mathbf{q}_{2}\left(\mathbf{c}^{0}\right), \ldots, \mathbf{q}_{n}\left(\mathbf{c}^{0}\right)\right], } \\
& \boldsymbol{\rho}\left(\mathbf{c}^{0}\right):=\left[\rho_{1}\left(\mathbf{c}^{0}\right), \rho_{2}\left(\mathbf{c}^{0}\right), \ldots, \rho_{n}\left(\mathbf{c}^{0}\right)\right]^{\top} \\
&=\left[\lambda_{1}\left(\mathbf{c}^{0}\right), \lambda_{2}\left(\mathbf{c}^{0}\right), \ldots, \lambda_{n}\left(\mathbf{c}^{0}\right)\right]^{\top} .
\end{aligned}
$$

For $k=0,1,2, \ldots$ until convergence do the following.
Step 1. Form $\left[J_{k}\right]_{i j}=\mathbf{p}_{i}\left(\mathbf{c}^{k}\right)^{\top} A_{j} \mathbf{p}_{i}\left(\mathbf{c}^{k}\right)$ for $1 \leq i, j \leq n$.

Step 2. Solve $\Delta \overline{\mathbf{c}}^{k}$ inexactly from the approximate Jacobian equation:

$$
J_{k} \Delta \overline{\mathbf{c}}^{k}+\boldsymbol{\rho}\left(\mathbf{c}^{k}\right)-\lambda^{*}=0
$$

such that

$$
\left\|J_{k} \Delta \overline{\mathbf{c}}^{k}+\boldsymbol{\rho}\left(\mathbf{c}^{k}\right)-\lambda^{*}\right\| \leq \bar{\eta}_{k}\left\|\rho\left(\mathbf{c}^{k}\right)-\lambda^{*}\right\|,
$$

where $\bar{\eta}_{0} \in(0,1)$ and

$$
\begin{aligned}
& \bar{\eta}_{k}:=\min \left\{\frac{\left\|\rho\left(\mathrm{c}^{k}\right)-\lambda^{*}\right\|^{\beta}}{\left\|\lambda^{*}\right\|^{\beta}}, \frac{\left\|\rho\left(\mathrm{c}^{k}\right)-\lambda^{*}\right\|^{\beta}}{\left\|\rho\left(\mathrm{c}^{k-1}\right)-\lambda^{*}\right\|^{\beta}}, \eta_{\max }\right\}, \\
& \beta \in(1,2], k=1,2, \ldots \text {. }
\end{aligned}
$$

Step 3. Set $\Delta \mathbf{c}^{k}=\Delta \overline{\mathbf{c}}^{k}$ and $\eta_{k}=\bar{\eta}_{k}$.

Step 4. Form the skew-symmetric matrix $\bar{Y}_{k}$ by (10) with $\mathrm{c}^{\text {new }}=\mathbf{c}^{k}+\Delta \mathbf{c}^{k}$.

Step 5. Compute matrix $\bar{P}_{k}:=\left[\mathbf{p}_{1}\left(\mathbf{c}^{k}+\Delta \mathbf{c}^{k}\right), \ldots, \mathbf{p}_{n}\left(\mathbf{c}^{k}+\Delta \mathbf{c}^{k}\right)\right]$ by solving (12).

Step 6. Compute $\boldsymbol{\rho}\left(\mathbf{c}^{k}+\Delta \mathbf{c}^{k}\right)=\left(\rho_{1}\left(\mathbf{c}^{k}+\Delta \mathbf{c}^{k}\right), \ldots, \rho_{n}\left(\mathbf{c}^{k}+\Delta \mathbf{c}^{k}\right)\right)^{\top}$ by (13).

Step 7. While $\left\|\rho\left(c^{k}+\Delta c^{k}\right)-\lambda^{*}\right\|>\left(1-\xi\left(1-\eta_{k}\right)\right)\left\|\rho\left(c^{k}\right)-\lambda^{*}\right\|$ do the following:

$$
\begin{aligned}
& \text { choose } \theta \in\left[\theta_{\min }, \theta_{\max }\right] \\
& \text { then update } \Delta \mathbf{c}^{k} \longleftarrow \theta \Delta \mathbf{c}^{k}, \eta_{k} \longleftarrow 1-\theta\left(1-\eta_{k}\right) .
\end{aligned}
$$

Step 8. Set $\mathbf{c}^{k+1}=\mathbf{c}^{k}+\Delta \mathbf{c}^{k}$. As Steps 4-6, compute, respectively, the new approximations $P_{k+1}:=\left[\mathbf{p}_{1}\left(\mathrm{c}^{k+1}\right), \ldots, \mathbf{p}_{n}\left(\mathbf{c}^{k+1}\right)\right]$ and $\boldsymbol{\rho}\left(\mathbf{c}^{k+1}\right):=\left(\rho_{1}\left(\mathbf{c}^{k+1}\right), \ldots, \rho_{n}\left(\mathbf{c}^{k+1}\right)\right)^{\top}$.

It is worth noting that if $\left\{\mathbf{c}^{k}\right\}$ has no accumulation point, $\left\{\mathbf{c}^{k}\right\}$ has one or more accumulation points and the Jacobian matrix is singular at each of them, or the vector $\Delta \overline{\mathbf{c}}^{k}$ computed by solving the Jacobian equation (18) is such that $\Delta \overline{\mathbf{c}}^{k}=0$, then our algorithm fails.

It is clear that if $\rho\left(\mathbf{c}^{k}\right)-\lambda^{*} \rightarrow 0$ as $k \rightarrow \infty$ and $\mathbf{c}^{*}$ is an accumulation point of $\left\{\mathbf{c}^{k}\right\}$, then $\boldsymbol{\rho}\left(\mathbf{c}^{*}\right)-\lambda^{*}=0$. Let $\left\{P_{k}\right\}$ be generated by Algorithm 1 (see Step 7) and define $E_{k}:=P_{k}-$ $Q^{*}$ for each $k=0,1, \ldots$. The following lemma is taken from [17, Lemma 2].

Lemma 2 (see [17]). For any $\mathbf{c}, \overline{\mathbf{c}} \in \mathbb{R}^{n}$, one has

$$
\|A(\mathbf{c})-A(\overline{\mathbf{c}})\| \leq L\|\mathbf{c}-\overline{\mathbf{c}}\|,
$$

where $L:=\left(\sum_{i=1}^{n}\left\|A_{i}\right\|^{2}\right)^{1 / 2}$.

Based on Lemma 2, the following lemma is a straightforward application of [9, Lemma 4]. 
Lemma 3. Assume that $\mathbf{c}^{*}$ is an accumulation point of $\left\{\mathbf{c}^{k}\right\}$. Let the given eigenvalues $\left\{\lambda_{i}^{*}\right\}_{i=1}^{n}$ be distinct. Then

$$
\left\|\boldsymbol{\rho}\left(\mathbf{c}^{k}\right)-\lambda^{*}\right\| \leq \sqrt{n} L\left\|\mathbf{c}^{k}-\mathbf{c}^{*}\right\|+\mu_{2}\left\|E_{k}\right\|
$$

for any $k \in \mathbb{N}$, where $\mu_{2}:=2 \sqrt{n} \cdot\left\|A\left(\mathbf{c}^{*}\right)\right\|$.

As shown in [18, Theorem 2.3], in the case when the given eigenvalues $\left\{\lambda_{i}^{*}\right\}_{i=1}^{n}$ are distinct, the eigenvalues of $A(\mathbf{c})$ are distinct too for any point $\mathbf{c}$ in some neighborhood of $\mathbf{c}^{*}$. It follows that the function $f(\cdot)$ defined in (3) is analytic in the same neighborhood. However, if $\mathbf{c}$ is not near the solution $\mathbf{c}^{*}$, the analyticity of the function $f(\cdot)$ cannot be guaranteed.

For any symmetric matrix $X \in \mathbb{R}^{n \times n}$, set $\Lambda(X):=$ $\operatorname{diag}\left(\lambda_{1}(X), \ldots, \lambda_{n}(X)\right)$, where $\lambda_{i}(X), i=1,2, \ldots, n$ are the eigenvalues of $X$. As proved by D. Sun and J. Sun [19, Theorem 4.7], $\Lambda(\cdot)$ is a strongly semismooth function. Based on this result, for any $c \in \mathbb{R}^{n}$ and $\varepsilon>0$, there exists $\delta>0$ sufficiently small such that

$$
\begin{aligned}
& \|\rho(\overline{\mathbf{c}})-\boldsymbol{\rho}(\mathbf{c})-J(\mathbf{c})(\overline{\mathbf{c}}-\mathbf{c})\| \\
& \quad \leq \varepsilon\|\overline{\mathbf{c}}-\mathbf{c}\|^{2}, \quad \text { for any } \overline{\mathbf{c}} \in \mathbf{B}(\mathbf{c}, \delta) .
\end{aligned}
$$

The following lemma says that the backtracking loop in Step 7 of Algorithm 1 terminates after a finite number of steps.

Lemma 4. There exists $\eta_{k}^{\min } \in[0,1)$ such that, for any $\eta_{k} \in$ $\left[\eta_{k}^{\min }, 1\right)$, there is $\Delta \mathbf{c}^{k}$ satisfying

$$
\left\|\boldsymbol{\rho}\left(\mathrm{c}^{k}+\Delta \mathrm{c}^{k}\right)-\lambda^{*}\right\| \leq\left[1-\xi\left(1-\eta_{k}\right)\right]\left\|\boldsymbol{\rho}\left(\mathrm{c}^{k}\right)-\lambda^{*}\right\| .
$$

Proof. By using the strong semismoothness of all eigenvalues of a real symmetric matrix [19], for any given $\varepsilon>0$, there exists $\delta>0$ sufficiently small such that

$$
\left\|\boldsymbol{\rho}\left(\mathbf{c}^{k}+\Delta \mathbf{c}\right)-\boldsymbol{\rho}\left(\mathbf{c}^{k}\right)-J_{k} \Delta \mathbf{c}\right\| \leq \varepsilon\|\Delta \mathbf{c}\|
$$

whenever $\|\Delta \mathrm{c}\| \leq \delta$. Choose

$$
\varepsilon=\frac{(1-\xi)\left(1-\bar{\eta}_{k}\right)\left\|\rho\left(\mathbf{c}^{k}\right)-\lambda^{*}\right\|}{\left\|\Delta \overline{\mathbf{c}}^{k}\right\|},
$$

and set

$$
\eta_{k}^{\min }:=\max \left\{\bar{\eta}_{k}, 1-\frac{\left(1-\bar{\eta}_{k}\right) \delta}{\left\|\Delta \overline{\mathbf{c}}^{k}\right\|}\right\}
$$

For any $\eta_{k} \in\left[\eta_{k}^{\min }, 1\right)$, let $\Delta \mathbf{c}^{k}:=\left(\left(1-\eta_{k}\right) /\left(1-\bar{\eta}_{k}\right)\right) \Delta \overline{\mathbf{c}}^{k}$. Then, by the definition of $\eta_{k}^{\min }$ given in (28), we have

$$
\begin{aligned}
\left\|\Delta \mathbf{c}^{k}\right\| & =\frac{1-\eta_{k}}{1-\bar{\eta}_{k}}\left\|\Delta \overline{\mathbf{c}}^{k}\right\| \leq \frac{1-\eta_{k}^{\text {min }}}{1-\bar{\eta}_{k}}\left\|\Delta \overline{\mathbf{c}}^{k}\right\| \\
& \leq \frac{\left(1-\bar{\eta}_{k}\right) \delta}{\left\|\Delta \overline{\mathbf{c}}^{k}\right\|} \cdot \frac{1}{1-\bar{\eta}_{k}}\left\|\Delta \overline{\mathbf{c}}^{k}\right\|=\delta .
\end{aligned}
$$

On the other hand, by (19), one gets that

$$
\begin{aligned}
& \left\|\rho\left(\mathrm{c}^{k}\right)-\lambda^{*}+J_{k} \Delta \mathbf{c}^{k}\right\| \\
& \leq \frac{\eta_{k}-\bar{\eta}_{k}}{1-\bar{\eta}_{k}}\left\|\rho\left(\mathrm{c}^{k}\right)-\lambda^{*}\right\|+\frac{1-\eta_{k}}{1-\bar{\eta}_{k}}\left\|\rho\left(\mathrm{c}^{k}\right)-\lambda^{*}+J_{k} \Delta \overline{\mathbf{c}}^{k}\right\| \\
& \leq \frac{\eta_{k}-\bar{\eta}_{k}}{1-\bar{\eta}_{k}}\left\|\rho\left(\mathrm{c}^{k}\right)-\lambda^{*}\right\|+\frac{1-\eta_{k}}{1-\bar{\eta}_{k}} \cdot \bar{\eta}_{k}\left\|\rho\left(\mathrm{c}^{k}\right)-\lambda^{*}\right\| \\
& =\eta_{k}\left\|\rho\left(\mathrm{c}^{k}\right)-\lambda^{*}\right\| .
\end{aligned}
$$

This together with (26) and (27) yields that

$$
\begin{aligned}
& \left\|\boldsymbol{\rho}\left(\mathbf{c}^{k}+\Delta \mathbf{c}^{k}\right)-\lambda^{*}\right\| \\
& \leq\left\|\rho\left(\mathbf{c}^{k}+\Delta \mathbf{c}^{k}\right)-\boldsymbol{\rho}\left(\mathbf{c}^{k}\right)-J_{k} \Delta \mathbf{c}^{k}\right\|+\left\|\rho\left(\mathbf{c}^{k}\right)-\lambda^{*}+J_{k} \Delta \mathbf{c}^{k}\right\| \\
& \leq \varepsilon\left\|\Delta \mathbf{c}^{k}\right\|+\eta_{k}\left\|\rho\left(\mathbf{c}^{k}\right)-\lambda^{*}\right\| \\
& =\varepsilon \frac{1-\eta_{k}}{1-\bar{\eta}_{k}}\left\|\Delta \overline{\mathbf{c}}^{k}\right\|+\eta_{k}\left\|\rho\left(\mathbf{c}^{k}\right)-\lambda^{*}\right\| \\
& =(1-\xi)\left(1-\eta_{k}\right)\left\|\rho\left(\mathbf{c}^{k}\right)-\lambda^{*}\right\|+\eta_{k}\left\|\rho\left(\mathbf{c}^{k}\right)-\lambda^{*}\right\| \\
& =\left[1-\xi\left(1-\eta_{k}\right)\right]\left\|\rho\left(\mathbf{c}^{k}\right)-\lambda^{*}\right\|,
\end{aligned}
$$

and the proof is completed.

Next, we give sufficient conditions for Algorithm 1 not to break down in the backtracking loop in Step 7.

Lemma 5. If $\rho\left(c^{k}\right)-\lambda^{*} \neq 0$ and there exists $\Gamma>0$ for which

$$
\left\|\Delta \mathbf{c}^{k}\right\| \leq \Gamma\left(1-\eta_{k}\right)\left\|\rho\left(\mathbf{c}^{k}\right)-\lambda^{*}\right\|
$$

then the backtracking loop terminates.

Proof. For constant $\Gamma$ in (32) and the given $\xi \in(0,1)$, choosing $\varepsilon=(1-\xi) / \Gamma$, there exists $\delta>0$ sufficiently small such that (26) holds whenever $\|\Delta \mathbf{c}\| \leq \delta$. We choose $\eta_{k} \in$ $\left[\bar{\eta}_{k}, 1\right]$ satisfying

$$
1-\eta_{k}<\frac{\delta}{\Gamma\left\|\rho\left(c^{k}\right)-\lambda^{*}\right\|}
$$

It follows that

$$
\left\|\Delta \mathbf{c}^{k}\right\| \leq \Gamma\left(1-\eta_{k}\right)\left\|\rho\left(\mathbf{c}^{k}\right)-\lambda^{*}\right\|<\delta,
$$

which gives $\mathbf{c}^{k}+\Delta \mathbf{c}^{k} \in \mathbf{B}\left(\mathbf{c}^{k}, \delta\right)$. Thus, we have

$$
\left\|\boldsymbol{\rho}\left(\mathbf{c}+\Delta \mathbf{c}^{k}\right)-\boldsymbol{\rho}\left(\mathbf{c}^{k}\right)-J_{k} \Delta \mathbf{c}^{k}\right\| \leq \frac{1-\xi}{\Gamma}\left\|\Delta \mathbf{c}^{k}\right\| .
$$


This together with (19) gives that

$$
\begin{aligned}
& \left\|\boldsymbol{\rho}\left(\mathbf{c}^{k}+\Delta \mathbf{c}^{k}\right)-\lambda^{*}\right\| \\
& \leq\left\|\boldsymbol{\rho}\left(\mathbf{c}^{k}\right)-\lambda^{*}+J_{k} \Delta \mathbf{c}^{k}\right\|+\left\|\boldsymbol{\rho}\left(\mathbf{c}^{k}+\Delta \mathbf{c}^{k}\right)-\boldsymbol{\rho}\left(\mathbf{c}^{k}\right)-J_{k} \Delta \mathbf{c}^{k}\right\| \\
& \leq \eta_{k}\left\|\boldsymbol{\rho}\left(\mathbf{c}^{k}\right)-\lambda^{*}\right\|+(1-\xi)\left(1-\eta_{k}\right)\left\|\boldsymbol{\rho}\left(\mathbf{c}^{k}\right)-\lambda^{*}\right\| \\
& =\left(1-\xi\left(1-\eta_{k}\right)\right)\left\|\boldsymbol{\rho}\left(\mathbf{c}^{k}\right)-\lambda^{*}\right\| .
\end{aligned}
$$

It follows that the backtracking loop terminates. This completes the proof.

The next lemma gives condition under which (32) is satisfied.

Lemma 6. Assume that the Jacobian matrix $J_{k}$ is invertible and set $M_{k}:=\left\|J_{k}^{-1}\right\|$. Then there exists $\Gamma$ such that (32) holds.

Proof. By using condition (19), one has that

$$
\begin{aligned}
& \left\|\Delta \mathbf{c}^{k}\right\| \\
& \quad \leq\left\|J_{k}^{-1}\right\|\left\|J_{k} \Delta \mathbf{c}^{k}\right\| \\
& \leq M_{k}\left(\left\|\rho\left(\mathbf{c}^{k}\right)-\lambda^{*}\right\|+\left\|\rho\left(\mathbf{c}^{k}\right)-\lambda^{*}+J_{k} \Delta \mathbf{c}^{k}\right\|\right) \\
& \leq M_{k}\left(1+\eta_{k}\right)\left\|\rho\left(\mathbf{c}^{k}\right)-\lambda^{*}\right\| \\
& \quad=M_{k} \frac{1+\eta_{k}}{1-\eta_{k}}\left(1-\eta_{k}\right)\left\|\rho\left(\mathbf{c}^{k}\right)-\lambda^{*}\right\| \\
& \quad \leq M_{k} \frac{1+\eta_{\max }}{1-\eta_{\max }}\left(1-\eta_{k}\right)\left\|\rho\left(\mathbf{c}^{k}\right)-\lambda^{*}\right\| .
\end{aligned}
$$
$\left.\eta_{\max }\right)$.

We finish the proof by taking $\Gamma:=M_{k}\left(1+\eta_{\max }\right) /(1-$

Lemmas 5 and 6 yield the result below.

Corollary 7. Assume that $\rho\left(\mathrm{c}^{k}\right)-\lambda^{*} \neq 0$ and $J_{k}$ are invertible. Set $M_{k}:=\left\|J_{k}^{-1}\right\|$ and $\Gamma:=M_{k}\left(1+\eta_{\max }\right) /\left(1-\eta_{\max }\right)$. Then, the backtracking loop in Step 7 of Algorithm 1 terminates with

$$
1-\eta_{k} \geq \min \left\{1-\bar{\eta}_{k}, \frac{\delta \theta_{\min }}{\Gamma\left\|\rho\left(\mathbf{c}^{k}\right)-\lambda^{*}\right\|}\right\},
$$

for any $\delta>0$ small enough such that (26) holds whenever $\|\Delta \mathrm{c}\| \leq \delta$.

Proof. Suppose that $\eta_{k}$ is the final value determined by the while-loop. If $\eta_{k}=\bar{\eta}_{k}$, then (38) is trivial. Assume that $\eta_{k} \neq \bar{\eta}_{k}$; that is, the body of the while-loop has been executed at least once. Denoting the penultimate value by $\eta_{k}^{-}$, then it follows from (33) that $1-\eta_{k}^{-} \geq \delta / \Gamma\left\|\rho\left(\mathrm{c}^{k}\right)-\lambda^{*}\right\|$. Thus, we have

$$
1-\eta_{k}=\theta\left(1-\eta_{k}^{-}\right) \geq \theta_{\min }\left(1-\eta_{k}^{-}\right) \geq \frac{\delta \theta_{\min }}{\Gamma\left\|\rho\left(\mathbf{c}^{k}\right)-\lambda^{*}\right\|} .
$$

This completes the proof.
Lemma 8. Assume that $\mathbf{c}^{*}$ is an accumulation point of $\left\{\mathbf{c}^{k}\right\}$ such that there exists a constant $\Gamma$ independent of $k$ for which

$$
\left\|\Delta \mathbf{c}^{k}\right\| \leq \Gamma\left(1-\eta_{k}\right)\left\|\rho\left(\mathrm{c}^{k}\right)-\lambda^{*}\right\|,
$$

whenever $\mathrm{c}^{k}$ is sufficiently near $\mathrm{c}^{*}$ and $k$ is sufficient large. Then $\mathrm{c}^{k} \rightarrow \mathrm{c}^{*}$ as $k \rightarrow \infty$.

Proof. Suppose that $\mathbf{c}^{k} \nrightarrow \mathbf{c}^{*}$. Then, there exists $\delta>0$ sufficiently small such that there are infinitely many $k$ for which $\mathbf{c}^{k} \notin \mathbf{B}\left(\mathbf{c}^{*}, \delta\right)$ and (40) hold whenever $\mathbf{c}^{k} \in \mathbf{B}\left(\mathbf{c}^{*}, \delta\right)$ for $k$ sufficiently large.

Since $\mathbf{c}^{*}$ is an accumulation point of $\left\{\mathbf{c}^{k}\right\}$, there exists subsequence $\left\{\mathbf{c}^{k_{i}}\right\} \subset\left\{\mathbf{c}^{k}\right\}$ such that $\mathbf{c}^{k_{i}} \in \mathbf{B}\left(\mathbf{c}^{*}, \delta / i\right)$ for $i$ sufficiently large. Choose $\ell_{i}>0$ satisfying $k_{i}+\ell_{i}<k_{i+1}$ and $\mathbf{c}^{k_{i}+\ell_{i}} \notin \mathbf{B}\left(\mathbf{c}^{*}, \delta\right)$. It follows that

$$
\begin{aligned}
& \left\|\rho\left(\mathrm{c}^{k_{i}+j-1}\right)-\lambda^{*}\right\| \\
& \leq \frac{1}{\Gamma\left(1-\eta_{k_{i}+j-1}\right)}\left(\left\|\rho\left(\mathrm{c}^{k_{i}+j-1}\right)-\lambda^{*}\right\|-\left\|\rho\left(\mathrm{c}^{k_{i}+j}\right)-\lambda^{*}\right\|\right), \\
& j=1, \ldots, \ell_{i} .
\end{aligned}
$$

Then, by (40), we have, for $i$ sufficiently large,

$$
\begin{aligned}
\frac{\delta}{2} & \leq\left\|\mathbf{c}^{k_{i}+\ell_{i}}-\mathbf{c}^{k_{i}}\right\| \\
& \leq \sum_{k=k_{i}}^{k_{i}+\ell_{i}-1}\left\|\Delta \mathbf{c}^{k}\right\| \leq \sum_{k=k_{i}}^{k_{i}+\ell_{i}-1} \Gamma\left(1-\eta_{k}\right)\left\|\rho\left(\mathbf{c}^{k}\right)-\lambda^{*}\right\| \\
& \leq \frac{\Gamma}{t}\left(\left\|\rho\left(\mathbf{c}^{k_{i}}\right)-\lambda^{*}\right\|-\left\|\rho\left(\mathbf{c}^{k_{i+1}}\right)-\lambda^{*}\right\|\right) .
\end{aligned}
$$

Note that $\mathrm{c}^{k_{i}} \rightarrow \mathrm{c}^{*}$ as $i \rightarrow \infty$. It follows that $\left\|\rho\left(\mathrm{c}^{k_{i}}\right)-\lambda^{*}\right\|-$ $\left\|\rho\left(\mathbf{c}^{k_{i+1}}\right)-\lambda^{*}\right\| \rightarrow 0$, which is a contradiction. Therefore, $\mathrm{c}^{k} \rightarrow \mathrm{c}^{*}$ as $k \rightarrow \infty$.

Lemma 9. Assume that $\mathbf{c}^{*}$ is an accumulation point of $\left\{\mathbf{c}^{k}\right\}$ such that $J\left(\mathbf{c}^{*}\right)$ is invertible. Set $M=\left\|J\left(\mathbf{c}^{*}\right)^{-1}\right\|$ and let $\delta_{1}$ be such that $0<\delta_{1} \leq 1 / 2 \mu_{1} M$, where $\mu_{1}:=2 n \cdot \max _{1 \leq j \leq n}\left\|A_{j}\right\|$. Suppose that $\left\|E_{k}\right\| \leq \delta_{1}$ for $k$ sufficiently large. Then, for all $k$ sufficiently large,

$$
\left\|J_{k}^{-1}\right\| \leq \frac{\left\|J\left(\mathbf{c}^{*}\right)^{-1}\right\|}{1-\mu_{1} \delta_{1}\left\|J\left(\mathbf{c}^{*}\right)^{-1}\right\|} \leq 2 M
$$

Proof. By the definitions of $\left[J_{k}\right]_{i j}$ and $\left[J\left(\mathbf{c}^{*}\right)\right]_{i j}$, for all $k$ sufficiently large,

$$
\begin{aligned}
& \left|\left[J_{k}\right]_{i j}-\left[J\left(\mathbf{c}^{*}\right)\right]_{i j}\right| \\
& \leq\left|\mathbf{p}_{i}\left(\mathbf{c}^{k}\right)^{\top} A_{j} \mathbf{p}_{i}\left(\mathbf{c}^{k}\right)-\mathbf{p}_{i}\left(\mathbf{c}^{k}\right)^{\top} A_{j} \mathbf{q}_{i}\left(\mathbf{c}^{*}\right)\right| \\
& \quad+\left|\mathbf{p}_{i}\left(\mathbf{c}^{k}\right)^{\top} A_{j} \mathbf{q}_{i}\left(\mathbf{c}^{*}\right)-\mathbf{q}_{i}\left(\mathbf{c}^{*}\right)^{\top} A_{j} \mathbf{q}_{i}\left(\mathbf{c}^{*}\right)\right|
\end{aligned}
$$




$$
\begin{aligned}
\leq & \left\|\mathbf{p}_{i}\left(\mathbf{c}^{k}\right)^{\top}\right\|\left\|A_{j}\right\|\left\|\mathbf{p}_{i}\left(\mathbf{c}^{k}\right)-\mathbf{q}_{i}\left(\mathbf{c}^{*}\right)\right\| \\
& +\left\|\mathbf{p}_{i}\left(\mathbf{c}^{k}\right)^{\top}-\mathbf{q}_{i}\left(\mathbf{c}^{*}\right)^{\top}\right\|\left\|A_{j}\right\|\left\|\mathbf{q}_{i}\left(\mathbf{c}^{*}\right)\right\| \\
= & 2\left\|A_{j}\right\|\left\|\mathbf{p}_{i}\left(\mathbf{c}^{k}\right)-\mathbf{q}_{i}\left(\mathbf{c}^{*}\right)\right\|, \quad 1 \leq i, j \leq n .
\end{aligned}
$$

Then, we have, for all $k$ sufficiently large,

$$
\begin{aligned}
\| J_{k}- & J\left(\mathbf{c}^{*}\right)\|\leq\| J_{k}-J\left(\mathbf{c}^{*}\right) \|_{F} \\
& \leq 2 n \cdot \max _{1 \leq j \leq n}\left\|A_{j}\right\| \cdot \max _{1 \leq i \leq n}\left\|\mathbf{p}_{i}\left(\mathbf{c}^{k}\right)-\mathbf{q}_{i}\left(\mathbf{c}^{*}\right)\right\| .
\end{aligned}
$$

Noting that $\mathbf{p}_{i}\left(\mathbf{c}^{k}\right)-\mathbf{q}_{i}\left(\mathbf{c}^{*}\right)$ is the $i$ th column of $E_{k}$, then $\left\|\mathbf{p}_{i}\left(\mathbf{c}^{k}\right)-\mathbf{q}_{i}\left(\mathbf{c}^{*}\right)\right\| \leq\left\|E_{k}\right\|$ for $i=1, \ldots, n$. So, for all $k$ sufficiently large,

$$
\left\|J\left(\mathbf{c}^{*}\right)^{-1}\right\|\left\|J_{k}-J\left(\mathbf{c}^{*}\right)\right\| \leq \mu_{1} \delta_{1} M \leq \frac{1}{2}<1 .
$$

It follows from Banach lemma that $J_{k}$ is invertible and $\left\|J_{k}^{-1}\right\| \leq 2 M$ for all $k$ sufficiently large. This completes the proof.

Lemmas 6, 8, and 9 yield the result below.

Corollary 10. Assume that $\mathbf{c}^{*}$ is an accumulation point of $\left\{\mathbf{c}^{k}\right\}$ such that $J\left(\mathbf{c}^{*}\right)$ is invertible. Set $M:=\left\|J\left(\mathbf{c}^{*}\right)^{-1}\right\|$ and let $\delta_{1}$ be determined by Lemma 9. Assume that $\left\|E_{k}\right\| \leq \delta_{1}$ for $k$ sufficiently large. Then $\mathrm{c}^{k} \rightarrow \mathrm{c}^{*}$ as $k \rightarrow \infty$.

Proof. By Lemma 9, we know $J_{k}$ is invertible and $\left\|J_{k}^{-1}\right\| \leq$ $2 M$ for all $k$ sufficiently large. From the proof of Lemma 6 , (40) holds for the constant $\Gamma=2 M\left(1+\eta_{\max }\right) /\left(1-\eta_{\max }\right)$ independent of $k$. Therefore, $c^{k} \rightarrow \mathbf{c}^{*}$ as $k \rightarrow \infty$ follows from Lemma 8.

Corollary 11. Assume that $\mathbf{c}^{*}$ is an accumulation point of $\left\{\mathbf{c}^{k}\right\}$ such that $J\left(\mathbf{c}^{*}\right)$ is invertible. Then, $\rho\left(\mathbf{c}^{k}\right)-\lambda^{*} \rightarrow 0$ as $k \rightarrow \infty$. Moreover, for all $k$ sufficiently large, one has $\eta_{k}=\bar{\eta}_{k}$.

Proof. If $\Delta \mathrm{c}^{k}$ is computed in the backtracking loop, the backtracking terminates with $\eta_{k}$ such that (38) holds. Since $c^{k} \rightarrow$ $\mathbf{c}^{*}$ by Corollary 10 , we have $\mathbf{c}^{k} \in \mathbf{B}\left(\mathbf{c}^{*}, \delta\right)$ for all $k$ sufficiently large. Thus, the series $\sum_{k=0}^{\infty}\left(1-\eta_{k}\right)$ is divergent. Then, we have

$$
\begin{aligned}
\prod_{1 \leq i<k}\left[1-\xi\left(1-\eta_{i}\right)\right] & \leq \prod_{1 \leq i<k} e^{-\xi\left(1-\eta_{i}\right)} \\
& =e^{-\xi \cdot \sum_{i=1}^{k-1}\left(1-\eta_{i}\right)} \longrightarrow 0, \quad k \longrightarrow \infty,
\end{aligned}
$$

and so

$$
\begin{aligned}
& \left\|\boldsymbol{\rho}\left(\mathrm{c}^{k}\right)-\lambda^{*}\right\| \\
& \leq\left[1-\xi\left(1-\eta_{k-1}\right)\right]\left\|\rho\left(\mathrm{c}^{k-1}\right)-\lambda^{*}\right\| \\
& \leq\left\|\boldsymbol{\rho}\left(\mathbf{c}^{0}\right)-\lambda^{*}\right\| \prod_{0 \leq i<k}\left[1-\xi\left(1-\eta_{i}\right)\right] \longrightarrow 0, \quad k \rightarrow \infty .
\end{aligned}
$$

This together with (38) yields that $\eta_{k}=\bar{\eta}_{k}$ for all $k$ sufficiently large.

Lemma 12. Assume that $\mathbf{c}^{*}$ is an accumulation point of $\left\{\mathbf{c}^{k}\right\}$ such that $J\left(\mathrm{c}^{*}\right)$ is invertible. Then, there exists $\delta_{2}>0$ such that

$$
\left\|\rho\left(c^{k}\right)-\lambda^{*}-J_{k}\left(c^{k}-c^{*}\right)\right\| \leq \mu_{3}\left\|E_{k}\right\|_{F}^{2}
$$

whenever $\mathbf{c}^{k} \in \mathbf{B}\left(\mathbf{c}^{*}, \delta_{2}\right)$ and $k$ sufficiently large, where $\mu_{3}:=$ $2 \max _{1 \leq i \leq n}\left|\lambda_{i}^{*}\right|$.

Proof. Set $H_{k}:=\mathrm{Q}\left(\mathrm{c}^{*}\right)^{\top} P_{k}-\mathrm{I}$. Then, we have, for all $k$ sufficiently large,

$$
\begin{aligned}
P_{k}^{\top} A\left(\mathbf{c}^{*}\right) P_{k} & =\left(\mathbf{I}+H_{k}\right)^{\top} \Lambda^{*}\left(\mathbf{I}+H_{k}\right) \\
& =\Lambda^{*}+\Lambda^{*} H_{k}+H_{k}^{\top} \Lambda^{*}+H_{k}^{\top} \Lambda^{*} H_{k} .
\end{aligned}
$$

It follows that, for all $k$ sufficiently large,

$$
\begin{aligned}
& \mathbf{p}_{i}\left(\mathbf{c}^{k}\right)^{\top} A\left(\mathbf{c}^{*}\right) \mathbf{p}_{i}\left(\mathbf{c}^{k}\right) \\
& \quad=\lambda_{i}^{*}+2 \lambda_{i}^{*}\left[H_{k}\right]_{i i}+\sum_{j=1}^{n} \lambda_{j}^{*}\left[H_{k}\right]_{i j}^{2}, \quad i=1,2, \ldots, n .
\end{aligned}
$$

Set

$$
v_{i}:=2 \lambda_{i}^{*}\left[H_{k}\right]_{i i}+\sum_{j=1}^{n} \lambda_{j}^{*}\left[H_{k}\right]_{i j}^{2}, \quad i=1,2, \ldots, n,
$$

and write $\mathbf{v}=\left(v_{1}, v_{2}, \ldots, v_{n}\right)^{\top}$. It follows that $J_{k} \mathbf{c}^{*}=\lambda^{*}-\mathbf{a}^{k}+$ $\mathbf{v}$, where

$$
\left[\mathbf{a}^{k}\right]_{i}=\mathbf{p}_{i}\left(\mathbf{c}^{k}\right)^{\top} A_{0} \mathbf{p}_{i}\left(\mathbf{c}^{k}\right), \quad i=1,2, \ldots, n
$$

In view of that $J_{k} \mathbf{c}^{k}=\boldsymbol{\rho}\left(\mathbf{c}^{k}\right)-\mathbf{a}^{k}$ for any $k \in \mathbb{N}$, one has that, for all $k$ sufficiently large,

$$
\mathbf{v}=\boldsymbol{\rho}\left(\mathbf{c}^{k}\right)-\lambda^{*}-J_{k}\left(\mathbf{c}^{k}-\mathbf{c}^{*}\right)
$$

Since

$$
\begin{aligned}
\mathbf{I}+H_{k} H_{k}^{\top}+H_{k} H_{k}^{\top} & =\left(\mathbf{I}+H_{k}\right)\left(\mathbf{I}+H_{k}\right)^{\top} \\
& =Q\left(\mathbf{c}^{*}\right)^{\top} P_{k} P_{k}^{\top} Q\left(\mathbf{c}^{*}\right)=\mathbf{I},
\end{aligned}
$$

we have

$$
\left[H_{k}\right]_{i i}=-\frac{1}{2} \sum_{j=1}^{n}\left[H_{k}\right]_{i j}^{2}, \quad i=1,2, \ldots, n .
$$


It follows from (52) that, for all $k$ sufficiently large,

$$
\begin{aligned}
v_{i}^{2} & =\left(2 \lambda_{i}^{*}\left[H_{k}\right]_{i i}+\sum_{j=1}^{n} \lambda_{j}^{*}\left[H_{k}\right]_{i j}^{2}\right)^{2} \\
& \leq 2\left[4\left(\lambda_{i}^{*}\right)^{2}\left[H_{k}\right]_{i i}^{2}+\left(\sum_{j=1}^{n} \lambda_{j}^{*}\left[H_{k}\right]_{i j}^{2}\right)^{2}\right] .
\end{aligned}
$$

Combining (56) with (57), one has that, for all $k$ sufficiently large,

$$
\begin{aligned}
\sum_{i=1}^{n} v_{i}^{2} \leq & 2\left[\sum_{i=1}^{n} 4\left(\lambda_{i}^{*}\right)^{2}\left[H_{k}\right]_{i i}^{2}+\sum_{i=1}^{n}\left(\sum_{j=1}^{n} \lambda_{j}^{*}\left[H_{k}\right]_{i j}^{2}\right)^{2}\right] \\
= & 2\left[\sum_{i=1}^{n}\left(\lambda_{i}^{*}\right)^{2}\left(\sum_{j=1}^{n}\left[H_{k}\right]_{i j}^{2}\right)^{2}+\sum_{i=1}^{n}\left(\sum_{j=1}^{n} \lambda_{j}^{*}\left[H_{k}\right]_{i j}^{2}\right)^{2}\right] \\
\leq & 2\left[\max _{1 \leq i \leq n}\left|\lambda_{i}^{*}\right|^{2} \cdot \sum_{i=1}^{n}\left(\sum_{j=1}^{n}\left[H_{k}\right]_{i j}^{2}\right)^{2}\right. \\
& \left.+\max _{1 \leq i \leq n}\left|\lambda_{i}^{*}\right|^{2} \cdot \sum_{i=1}^{n}\left(\sum_{j=1}^{n}\left[H_{k}\right]_{i j}^{2}\right)^{2}\right] \\
\leq & 4 \max _{1 \leq i \leq n}\left|\lambda_{i}^{*}\right|^{2} \cdot\left(\sum_{i=1}^{n} \sum_{j=1}^{n}\left[H_{k}\right]_{i j}^{2}\right)^{2} \\
= & 4 \max _{1 \leq i \leq n}\left|\lambda_{i}^{*}\right|^{2} \cdot\left\|H_{k}\right\|_{F}^{4},
\end{aligned}
$$

which gives

$$
\|\mathbf{v}\| \leq 2 \max _{1 \leq i \leq n}\left|\lambda_{i}^{*}\right| \cdot\left\|H_{k}\right\|_{F}^{2}
$$

Note that,

$$
\left\|H_{k}\right\|_{F}=\left\|Q\left(\mathbf{c}^{*}\right)^{\top} P_{k}-\mathbf{I}\right\|_{F}=\left\|P_{k}-Q\left(\mathbf{c}^{*}\right)\right\|_{F}=\left\|E_{k}\right\|_{F} .
$$

Therefore, we obtain from (54) that (49) holds for all $k$ sufficiently large. This completes the proof.

Lemma 13. Assume that $\mathbf{c}^{*}$ is an accumulation point of $\left\{\mathbf{c}^{k}\right\}$ such that $J\left(\mathrm{c}^{*}\right)$ is invertible. Then, there exist $\delta_{3}>0, \delta_{4} \in$ $\left(0, \delta_{1}\right]$ sufficiently small such that $\left\|E_{k}\right\| \leq \delta_{4}$ and

$$
\left\|\mathbf{c}^{k+1}-\mathbf{c}^{*}\right\| \leq 2 M\left(\mu_{3}\left\|E_{k}\right\|_{F}^{2}+\left(\frac{2}{\mu_{3}}\right)^{\beta}\left\|\rho\left(\mathbf{c}^{k}\right)-\lambda^{*}\right\|^{\beta}\right)
$$

whenever $\mathbf{c}^{k} \in \mathbf{B}\left(\mathbf{c}^{*}, \delta_{3}\right)$ and $k$ sufficiently large, where $\delta_{1}$ is determined by Lemma 9.

Proof. Thanks to Corollary 11, we have $\eta_{k}=\bar{\eta}_{k}$ and $\Delta \mathbf{c}^{k}=\Delta \overline{\mathbf{c}}^{k}$ for all $k$ sufficiently large. Thus, the Jacobian equation (18) is equivalent to, for all $k$ sufficiently large,

$$
J_{k} \mathbf{c}^{k+1}-\lambda^{*}+\mathbf{a}^{k}=0 .
$$

Assume that the residual of this approximate Jacobian equation is defined by $\mathbf{r}^{k}$; that is, for all $k$ sufficiently large,

$$
\mathbf{r}^{k}=J_{k} \mathbf{c}^{k+1}-\lambda^{*}+\mathbf{a}^{k}
$$

This together with $J_{k} \mathbf{c}^{*}-\lambda^{*}+\mathbf{a}^{k}=\mathbf{v}$ gives, for all $k$ sufficiently large,

$$
J_{k}\left(\mathbf{c}^{*}-\mathbf{c}^{k+1}\right)=\mathbf{v}-\mathbf{r}^{k} .
$$

By (19), Lemmas 9 and 12, we obtain, for all $k$ sufficiently large,

$$
\begin{aligned}
\left\|\mathbf{c}^{k+1}-\mathbf{c}^{*}\right\| & \leq\left\|J_{k}^{-1}\right\|\left(\|\mathbf{v}\|+\left\|\mathbf{r}^{k}\right\|\right) \\
& \leq 2 M\left(\mu_{3}\left\|E_{k}\right\|_{F}^{2}+\frac{\left\|\boldsymbol{\rho}\left(\mathbf{c}^{k}\right)-\lambda^{*}\right\|^{\beta+1}}{\left\|\lambda^{*}\right\|^{\beta}}\right) .
\end{aligned}
$$

It follows from Lemma 3 that

$$
\left\|\rho\left(\mathbf{c}^{k}\right)-\lambda^{*}\right\| \leq \sqrt{n} L\left\|\mathbf{c}^{k}-\mathbf{c}^{*}\right\|+\mu_{2}\left\|E_{k}\right\|, \quad \forall k \in \mathbb{N} .
$$

Thus, we can choose $\delta_{3}>0$ and $0<\delta_{4} \leq \delta_{1}$ sufficiently small such that

$$
\sqrt{n} L \delta_{3}+\mu_{2} \delta_{4} \leq 1
$$

whenever $\left\|\mathbf{c}^{k}-\mathbf{c}^{*}\right\| \leq \delta_{3}$ and $\left\|E_{k}\right\|_{F} \leq \delta_{4}$. Therefore, combining this with the definition of $\mu_{3}$ given in Lemma 12, (61) follows.

Lemma 14 (see [6]). There exist two positive numbers $\delta_{5}$ and $\omega_{1}$ such that, for any orthogonal matrix $P$ with $\left\|P-Q\left(\mathbf{c}^{*}\right)\right\|<$ $\delta_{5}$, the skew-symmetric $X$ defined by $e^{X}:=P^{\top} Q\left(c^{*}\right)$ satisfies $\|X\| \leq \omega_{1}\left\|P-Q\left(\mathbf{c}^{*}\right)\right\|$.

Based on Lemma 14, by using the similar arguments in the proof of [10, Lemma 5], we can obtain the following lemma. If we write $e^{X_{k}}:=P_{k}^{\top} Q^{*}$, then there exists $C>0$ such that $\left\|Y_{k}\right\| \leq C\left(\left\|\mathbf{c}^{k+1}-\mathbf{c}^{*}\right\|+\left\|X_{k}\right\|\right)$.

Lemma 15. Suppose that the given eigenvalues $\left\{\lambda_{i}^{*}\right\}_{i=1}^{n}$ are distinct and the Jacobian matrix $J\left(\mathbf{c}^{*}\right)$ is invertible. Then, there exist $\omega_{2}>0$ and $0<\delta_{6}<\min \left\{\delta_{5}, 1 /\left(1+\omega_{1}\right) C\right\}$ such that, for $k$ sufficiently large, if $\left\|c^{k+1}-c^{*}\right\| \leq \delta_{6}$ and $\left\|E_{k}\right\| \leq \delta_{6}$, then

$$
\left\|E_{k+1}\right\| \leq \omega_{2}\left(\left\|\mathbf{c}^{k+1}-\mathbf{c}^{*}\right\|+\left\|E_{k}\right\|^{2}\right),
$$

where $\omega_{1}$ is determined by Lemma 14.

In order to prove our global convergence result for Algorithm 1, we introduce some notations. Let $\zeta_{1}:=2(\sqrt{n} L+$ $\left.\mu_{2}\right) / \mu_{3}$ and $\zeta_{2}:=2 M\left(\mu_{3}+\zeta_{1}^{\beta}\right)$. Set

$$
\begin{aligned}
\tau:=\min \left\{1,\left(2 M\left(\mu_{3}+\zeta_{1}^{\beta}\right)\right)^{-(1 /(\beta-1))}\right. \\
\left.\times\left(\omega_{2}\left(\zeta_{2}+1\right)\right)^{-(1 /(\beta-1))}\right\}, \\
\delta:=\min \left\{\tau, \delta_{2}, \delta_{3}, \delta_{4}, \delta_{6}\right\} .
\end{aligned}
$$

Our main global convergence result is as follows. 
Theorem 16. Assume that $\left\{\mathbf{c}^{k}\right\}$ is generated by Algorithm 1 . Suppose that the given eigenvalues $\left\{\lambda_{i}^{*}\right\}_{i=1}^{n}$ are distinct and $\mathbf{c}^{*}$ is an accumulation point of $\left\{\mathbf{c}^{k}\right\}$ such that $J\left(\mathbf{c}^{*}\right)$ is invertible. Then, $\rho\left(\mathbf{c}^{k}\right)-\lambda^{*} \rightarrow 0$ and $\mathbf{c}^{k} \rightarrow \mathrm{c}^{*}$ as $k \rightarrow \infty$. Moreover, the convergence is of $R$-order $\beta$.

Proof. It follows immediately from Corollaries 10 and 11 that $\boldsymbol{\rho}\left(\mathbf{c}^{k}\right)-\boldsymbol{\lambda}^{*} \rightarrow 0$ and $\mathbf{c}^{k} \rightarrow \mathbf{c}^{*}$ as $k \rightarrow \infty$. For the $\tau$ given in (69), there exists $k_{0}$ sufficiently large such that $\left\|\mathbf{c}^{k_{0}}-\mathrm{c}^{*}\right\| \leq \tau$ and $\left\|E_{k_{0}}\right\| \leq \tau$. Set $\gamma:=\delta / \tau$, where $\delta$ and $\tau$ are given in (70) and (69), respectively. Then, $\gamma \leq 1$. We will show that, for all $k \geq k_{0}$ sufficiently large,

$$
\begin{gathered}
\left\|\mathbf{c}^{k}-\mathbf{c}^{*}\right\| \leq \tau \cdot \gamma^{\beta^{k-k_{0}}}, \\
\left\|E_{k}\right\| \leq \tau \cdot \gamma^{\beta^{k-k_{0}}} .
\end{gathered}
$$

Suppose that (71) and (72) hold for some $k=\ell \geq k_{0}$. Consider the case $k=\ell+1$. Thanks to Lemma 3, we have, for all $\ell \geq k_{0}$,

$$
\begin{aligned}
\left\|\rho\left(c^{\ell}\right)-\lambda^{*}\right\| & \leq \sqrt{n} L\left\|c^{\ell}-c^{*}\right\|+\mu_{2}\left\|E_{\ell}\right\| \\
& \leq \sqrt{n} L \tau \cdot \gamma^{\beta^{\ell-k_{0}}}+\mu_{2} \tau \cdot \gamma^{\beta^{\ell-k_{0}}} \\
& =\left(\sqrt{n} L+\mu_{2}\right) \tau \cdot \gamma^{\beta^{\ell-k_{0}}} .
\end{aligned}
$$

Then, by using Lemma 13 , one has that, for all $\ell \geq k_{0}$,

$$
\begin{aligned}
& \left\|\mathbf{c}^{\ell+1}-\mathbf{c}^{*}\right\| \\
& \leq 2 M\left(\mu_{3}\left\|E_{\ell}\right\|_{F}^{2}+\left(\frac{2}{\mu_{3}}\right)^{\beta}\left\|\rho\left(\mathbf{c}^{\ell}\right)-\lambda^{*}\right\|\right) \\
& \leq 2 M \mu_{3} \tau^{2}\left(\gamma^{\beta^{\ell-k_{0}}}\right)^{2}+2 M\left(\frac{2}{\mu_{3}}\right)^{\beta}\left(\sqrt{n} L+\mu_{3}\right)^{\beta} \tau^{\beta} \gamma^{\beta^{\ell-k_{0}+1}} \\
& \leq 2 M\left[\mu_{3}+\left(\frac{2\left(\sqrt{n} L+\mu_{2}\right)}{\mu_{3}}\right)^{\beta}\right] \tau^{\beta} \gamma^{\beta^{\ell-k_{0}+1}} \\
& \leq \tau \gamma^{\beta^{\ell-k_{0}+1}}
\end{aligned}
$$

where the last inequality follows from the definition of $\tau$ in (69). By Lemma 15, we have, for all $\ell \geq k_{0}$,

$$
\begin{aligned}
\left\|E_{\ell+1}\right\| & \leq \omega_{2}\left(\left\|\mathbf{c}^{\ell+1}-\mathbf{c}^{*}\right\|+\left\|E_{\ell}\right\|^{2}\right) \\
& \leq 2 M \omega_{2}\left(\mu_{3}+\zeta_{1}^{\beta}\right) \tau^{\beta} \gamma^{\beta^{\ell-k_{0}+1}}+\omega_{2} \tau^{2}\left(\gamma^{\beta^{\ell-k_{0}}}\right)^{2} \\
& \leq \omega\left(2 M\left(\mu_{3}+\zeta_{1}^{\beta}\right)+1\right) \tau^{\beta} \cdot \gamma^{\beta^{\ell-k_{0}+1}} \\
& =\omega_{2}\left(\zeta_{2}+1\right) \tau^{\beta} \cdot \gamma^{\beta^{\ell-k_{0}+1}} \leq \tau \cdot \gamma^{\beta^{\ell-k_{0}+1}} .
\end{aligned}
$$

Therefore, we conclude that (71) and (72) hold for all $k \geq k_{0}$. Moreover, we see from (71) that $c^{k}$ converges to $c^{*}$ with $\mathrm{R}$ order $\beta$. This completes the proof.
If $\left\{\mathbf{c}^{k}\right\}$ generated by Algorithm 1 converges to a solution at which the Jacobian matrix is invertible, then the ultimate rate of convergence is governed by the choices of the $\eta_{k}(k=$ $0,1, \ldots)$ as in the local theory of [9].

\section{Numerical Examples}

In this section, we illustrate the effectiveness of Algorithm 1 in solving IEP on three examples. The tests were carried out in MATLAB 7.0 running on a PC Intel Pentium P6200 of $2.13 \mathrm{GHz}$ CPU.

The given parameters used in our algorithm were $\eta_{0}=$ $0.5, \eta_{\max }=0.9, \xi=10^{-4}, \theta_{\min }=0.1$, and $\theta_{\max }=0.9$. In the while loop, we choose $\theta \in\left[\theta_{\min }, \theta_{\max }\right]$ to minimize $\| \rho\left(\mathbf{c}^{k}+\right.$ $\left.\theta \Delta \mathbf{c}^{k}\right)-\lambda^{*} \|$ if 80 iterations of the backtracking loop fail to produce the sufficient decrease in $\left\|\rho(\mathbf{c})-\lambda^{*}\right\|$.

Linear systems (14) and (19) are all solved iteratively by the QMR method [20] using the MATLAB qmr function. In order to guarantee the orthogonality of $P^{\text {new }}$ in (14), this system is solved up to machine precision eps $\left(\approx 2.2 \times 10^{-16}\right)$. The inner loop stopping tolerance for (18) is given by (20). The stopping criterion of the outer iteration in our algorithm is

$$
\left\|P_{k}^{\top} A\left(\mathbf{c}^{k}\right) P_{k}-\Lambda_{*}\right\|_{F} \leq 10^{-10}
$$

Example 1. This is an inverse Toeplitz eigenvalue problem (see [2] for more details on this inverse problem) with distinct eigenvalues. The basis matrices $\left\{A_{i}\right\}_{i=1}^{5}$ are given as follows:

$$
\begin{array}{ll}
A_{1}=\left[\begin{array}{lllll}
1 & 0 & 0 & 0 & 0 \\
0 & 1 & 0 & 0 & 0 \\
0 & 0 & 1 & 0 & 0 \\
0 & 0 & 0 & 1 & 0 \\
0 & 0 & 0 & 0 & 1
\end{array}\right], & A_{2}=\left[\begin{array}{lllll}
0 & 1 & 0 & 0 & 0 \\
1 & 0 & 1 & 0 & 0 \\
0 & 1 & 0 & 1 & 0 \\
0 & 0 & 1 & 0 & 1 \\
0 & 0 & 0 & 1 & 0
\end{array}\right], \\
A_{3}=\left[\begin{array}{lllll}
0 & 0 & 1 & 0 & 0 \\
0 & 0 & 0 & 1 & 0 \\
1 & 0 & 0 & 0 & 1 \\
0 & 1 & 0 & 0 & 0 \\
0 & 0 & 1 & 0 & 0
\end{array}\right], & A_{4}=\left[\begin{array}{lllll}
0 & 0 & 0 & 1 & 0 \\
0 & 0 & 0 & 0 & 1 \\
0 & 0 & 0 & 0 & 0 \\
1 & 0 & 0 & 0 & 0 \\
0 & 1 & 0 & 0 & 0
\end{array}\right],
\end{array}
$$

$$
A_{5}=\left[\begin{array}{lllll}
0 & 0 & 0 & 0 & 1 \\
0 & 0 & 0 & 0 & 0 \\
0 & 0 & 0 & 0 & 0 \\
0 & 0 & 0 & 0 & 0 \\
1 & 0 & 0 & 0 & 0
\end{array}\right] .
$$

The given real eigenvalues and a solution, respectively, are

$$
\begin{gathered}
\lambda^{*}=(-5.2361,-1.5876,-0.7639,-0.5555,18.1431)^{\top}, \\
\mathbf{c}^{*}=(2,3,4,5,6)^{\top} .
\end{gathered}
$$


TABLE 1: Numerical results for Example 1.

\begin{tabular}{|c|c|c|c|c|}
\hline$c^{0}$ & & $\beta=1.5$ & $\beta=1.8$ & $\beta=2.0$ \\
\hline \multirow{5}{*}{ (a) } & \multirow{3}{*}{ errs } & $1.0659 e-02$ & $1.0659 e-02$ & $1.0659 e-02$ \\
\hline & & $1.1323 e-05$ & $1.1323 e-05$ & $1.1323 e-05$ \\
\hline & & $8.0567 e-12$ & $8.0567 e-12$ & $8.0567 e-12$ \\
\hline & ite. & 6 & 6 & 6 \\
\hline & $c^{*}$ & \multicolumn{3}{|c|}{$(2.0000,3.2926,3.4471,4.9014,6.5529)^{\top}$} \\
\hline \multirow{5}{*}{ (b) } & \multirow{3}{*}{ errs } & $3.8846 e-03$ & $3.8846 e-03$ & $3.8846 e-03$ \\
\hline & & $1.2491 e-06$ & $1.2491 e-06$ & $1.2491 e-06$ \\
\hline & & $7.9990 e-14$ & $7.9990 e-14$ & $7.9990 e-14$ \\
\hline & ite. & 9 & 9 & 9 \\
\hline & $c^{*}$ & & $(2,3,4,5,6)^{\top}$ & \\
\hline \multirow{5}{*}{ (c) } & \multirow{3}{*}{ errs } & $5.0389 e-05$ & $5.0389 e-05$ & $5.0389 e-05$ \\
\hline & & $3.5838 e-10$ & $3.5838 e-10$ & $3.5838 e-10$ \\
\hline & & $5.0337 e-15$ & $5.0337 e-15$ & $5.0337 e-15$ \\
\hline & ite. & 13 & 13 & 13 \\
\hline & $\mathrm{c}^{*}$ & \multicolumn{3}{|c|}{$(2.0000,3.2926,3.4471,4.9014,6.5529)^{\top}$} \\
\hline \multirow{5}{*}{ (d) } & \multirow{3}{*}{ errs } & $1.3806 e-04$ & $1.3806 e-04$ & $1.3806 e-04$ \\
\hline & & $6.4118 e-09$ & $6.4118 e-09$ & $6.4118 e-09$ \\
\hline & & $9.8493 e-15$ & $9.8493 e-15$ & $9.8493 e-15$ \\
\hline & ite. & 75 & 75 & 75 \\
\hline & $c^{*}$ & \multicolumn{3}{|c|}{$(2.0000,3.2926,3.4471,4.9014,6.5529)^{\top}$} \\
\hline \multirow{5}{*}{ (e) } & \multirow{3}{*}{ errs } & $1.2028 e-04$ & $7.7624 e-03$ & $7.7624 e-03$ \\
\hline & & $3.8608 e-10$ & $6.1243 e-06$ & $6.1243 e-06$ \\
\hline & & $5.3680 e-15$ & $2.1634 e-12$ & $2.1634 e-12$ \\
\hline & ite. & 13 & 5 & 5 \\
\hline & $c^{*}$ & & $(2,3,4,5,6)^{\top}$ & \\
\hline
\end{tabular}

In Table 1, we report our numerical results for various starting points:

$$
\begin{gathered}
\text { (a) } c^{0}=(1,2,3,4,5)^{\top}, \\
\text { (b) } c^{0}=(1,5,10,15,20)^{\top}, \\
\text { (c) } c^{0}=(11,12,13,14,15)^{\top}, \\
\text { (d) } c^{0}=(21,38,46,63,81)^{\top},
\end{gathered}
$$$$
\text { (e) } \quad \mathbf{c}^{0}=(101,112,123,134,145)^{\top} \text {, }
$$

where $\mathbf{c}^{0}$, errs, ite. and $\mathbf{c}^{*}$ stand for the starting point, the error value of the left-hand side of (76) for the last three iterates of the algorithm, the number of outer iteration, and the accumulation point corresponding to the starting point.

Example 2. This is a Toeplitz-plus-Hankel inverse eigenvalue problem (see [1] for more details on this inverse problem) with distinct eigenvalues. The basis matrices $\left\{A_{i}\right\}_{i=1}^{7}$ are given as follows:

$$
A_{1}=\left[\begin{array}{ccccccc}
-1 & 0 & 0 & 0 & 0 & 0 & 0 \\
0 & 1 & 0 & 0 & 0 & 0 & 0 \\
0 & 0 & 1 & 0 & 0 & 0 & 0 \\
0 & 0 & 0 & 1 & 0 & 0 & 0 \\
0 & 0 & 0 & 0 & 1 & 0 & 0 \\
0 & 0 & 0 & 0 & 0 & 1 & 0 \\
0 & 0 & 0 & 0 & 0 & 0 & 1
\end{array}\right],
$$$$
A_{2}=\left[\begin{array}{ccccccc}
0 & -1 & 0 & 0 & 0 & 0 & 0 \\
-1 & 0 & 1 & 0 & 0 & 0 & 0 \\
0 & 1 & 0 & 1 & 0 & 0 & 0 \\
0 & 0 & 1 & 0 & 1 & 0 & 0 \\
0 & 0 & 0 & 1 & 0 & 1 & 0 \\
0 & 0 & 0 & 0 & 1 & 0 & 1 \\
0 & 0 & 0 & 0 & 0 & 1 & 0
\end{array}\right],
$$$$
A_{3}=\left[\begin{array}{ccccccc}
0 & 0 & -1 & 0 & 0 & 0 & 0 \\
0 & -2 & 0 & 1 & 0 & 0 & 0 \\
-1 & 0 & 0 & 0 & 1 & 0 & 0 \\
0 & 1 & 0 & 0 & 0 & 1 & 0 \\
0 & 0 & 1 & 0 & 0 & 0 & 1 \\
0 & 0 & 0 & 1 & 0 & 0 & 0 \\
0 & 0 & 0 & 0 & 1 & 0 & 0
\end{array}\right],
$$$$
A_{4}=\left[\begin{array}{ccccccc}
0 & 0 & 0 & -1 & 0 & 0 & 0 \\
0 & 0 & -2 & 0 & 1 & 0 & 0 \\
0 & -2 & 0 & 0 & 0 & 1 & 0 \\
-1 & 0 & 0 & 0 & 0 & 0 & 1 \\
0 & 1 & 0 & 0 & 0 & 0 & 0 \\
0 & 0 & 1 & 0 & 0 & 0 & 0 \\
0 & 0 & 0 & 1 & 0 & 0 & 0
\end{array}\right],
$$

$$
A_{5}=\left[\begin{array}{ccccccc}
0 & 0 & 0 & 0 & -1 & 0 & 0 \\
0 & 0 & 0 & -2 & 0 & 1 & 0 \\
0 & 0 & -2 & 0 & 0 & 0 & 1 \\
0 & -2 & 0 & 0 & 0 & 0 & 0 \\
-1 & 0 & 0 & 0 & 0 & 0 & 0 \\
0 & 1 & 0 & 0 & 0 & 0 & 0 \\
0 & 0 & 1 & 0 & 0 & 0 & 0
\end{array}\right],
$$$$
A_{6}=\left[\begin{array}{ccccccc}
0 & 0 & 0 & 0 & 0 & -1 & 0 \\
0 & 0 & 0 & 0 & -2 & 0 & 1 \\
0 & 0 & 0 & -2 & 0 & 0 & 0 \\
0 & 0 & -2 & 0 & 0 & 0 & 0 \\
0 & -2 & 0 & 0 & 0 & 0 & 0 \\
-1 & 0 & 0 & 0 & 0 & 0 & 0 \\
0 & 1 & 0 & 0 & 0 & 0 & 0
\end{array}\right],
$$

$$
A_{7}=\left[\begin{array}{ccccccc}
0 & 0 & 0 & 0 & 0 & 0 & -1 \\
0 & 0 & 0 & 0 & 0 & -2 & 0 \\
0 & 0 & 0 & 0 & -2 & 0 & 0 \\
0 & 0 & 0 & -2 & 0 & 0 & 0 \\
0 & 0 & -2 & 0 & 0 & 0 & 0 \\
0 & -2 & 0 & 0 & 0 & 0 & 0 \\
-1 & 0 & 0 & 0 & 0 & 0 & 0
\end{array}\right] .
$$


The given real eigenvalues are

$$
\begin{aligned}
\lambda^{*}=( & -35.4513,-13.6805,-9.5675, \\
& -8.5489,8.7666,11.8220,26596)^{\top},
\end{aligned}
$$

and $\mathbf{c}^{*}=(2,3,4,5,6,7,8)^{\top}$ is a solution. In Table 2 , we report our numerical results for various starting points:

$$
\begin{gathered}
\text { (a) } c^{0}=(1,2,3,4,5,6,7)^{\top}, \\
\text { (b) } c^{0}=(1,3,5,7,9,11,13)^{\top}, \\
\text { (c) } c^{0}=(11,13,15,17,19,21,23)^{\top}, \\
\text { (d) } c^{0}=(50,52,56,58,62,65,68)^{\top}, \\
\text { (e) } c^{0}=(101,102,103,104,106,108,110)^{\top} .
\end{gathered}
$$

Example 3 (see $[10])$. Given $B=\left[b_{i j}\right]_{8 \times 8}:=\mathbf{I}+V V^{\top}$, where

$$
V=\left[\begin{array}{ccccc}
1 & -1 & -3 & -5 & -6 \\
1 & 1 & -2 & -5 & -17 \\
1 & -1 & -1 & 5 & 18 \\
1 & 1 & 1 & 2 & 0 \\
1 & -1 & 2 & 0 & 1 \\
1 & 1 & 3 & 0 & -1 \\
2.5 & 0.2 & 0.3 & 0.5 & 0.6 \\
2 & -0.2 & 0.3 & 0.5 & 0.8
\end{array}\right]_{8 \times 5}
$$

the basis matrices $\left\{A_{k}\right\}_{k=1}^{8}$ are defined from $B$ as follows:

$$
A_{k}=b_{k k} e_{k} e_{k}^{\top}+\sum_{j=1}^{k-1} b_{k j}\left(e_{k} e_{k}^{\top}+e_{j} e_{k}^{\top}\right), \quad k=1,2, \ldots, 8
$$

where $e_{k}$ is the $k$ th column of the identity matrix I. The given real eigenvalues and a solution, respectively, are

$$
\begin{gathered}
\lambda^{*}=(1.0 e+04) \times(-1.1672,-0.3139,-0.0140,-0.0008, \\
0.0003,0.0075,0.0327,1.7372)^{\top}
\end{gathered}
$$

\begin{tabular}{|c|c|c|c|}
\hline$c^{0}$ & $\beta=1.5$ & $\beta=1.8$ & $\beta=2.0$ \\
\hline \multirow[b]{3}{*}{ (a) } & $8.2730 e-03$ & $8.2730 e-03$ & $8.2730 e-03$ \\
\hline & $2.2866 e-05$ & $2.2866 e-05$ & $2.2866 e-05$ \\
\hline & $5.5845 e-11$ & $5.5845 e-11$ & $5.5845 e-11$ \\
\hline \multirow{2}{*}{$\begin{array}{l}\text { ite. } \\
c^{*}\end{array}$} & 6 & 6 & 6 \\
\hline & \multicolumn{3}{|c|}{$(2,3,4,5,6,7,8)^{\top}$} \\
\hline \multirow{3}{*}{ (b) } & $8.2129 e-03$ & $4.0190 e-03$ & $1.7235 e-04$ \\
\hline & $2.0027 e-05$ & $1.5988 e-06$ & $3.7839 e-09$ \\
\hline & $5.0828 e-11$ & $2.8276 e-13$ & $1.9386 e-14$ \\
\hline ite. & 7 & 7 & 7 \\
\hline$c^{*}$ & \multicolumn{3}{|c|}{$(2.8703,2.0639,4.9434,4.1053,6.7530,6.3287,8.4794)^{\top}$} \\
\hline \multirow[b]{3}{*}{ (c) } & $3.4941 e-03$ & $3.4941 e-03$ & $3.4941 e-03$ \\
\hline & $2.5608 e-06$ & $2.5608 e-06$ & $2.5608 e-06$ \\
\hline & $6.7550 e-13$ & $6.7550 e-13$ & $6.7550 e-13$ \\
\hline ite. & 11 & 11 & 11 \\
\hline$c^{*}$ & \multicolumn{3}{|c|}{$(2.8703,2.0639,4.9434,4.1053,6.7530,6.3287,8.4794)^{\top}$} \\
\hline \multirow[b]{3}{*}{ (d) } & $2.9914 e-04$ & $2.9914 e-04$ & $2.9914 e-04$ \\
\hline & $8.2616 e-09$ & $8.2616 e-09$ & $8.2616 e-09$ \\
\hline & $1.8946 e-14$ & $1.8946 e-14$ & $1.8946 e-14$ \\
\hline ite. & 13 & 13 & 13 \\
\hline$c^{*}$ & \multicolumn{3}{|c|}{$(1.1787,-0.0035,2.0401,1.6976,5.3794,6.2068,8.5273)^{\top}$} \\
\hline \multirow{5}{*}{ (e) } & $2.1008 e-04$ & $2.1008 e-04$ & $2.1008 e-04$ \\
\hline & $1.5703 e-08$ & $1.5703 e-08$ & $1.5703 e-08$ \\
\hline & $2.2331 e-14$ & $2.2331 e-14$ & $2.2331 e-14$ \\
\hline & 13 & 13 & 13 \\
\hline & \multicolumn{3}{|c|}{$(2.8703,2.0639,4.9434,4.1053,6.7530,6.3287,8.4794)^{\top}$} \\
\hline
\end{tabular}

and $\mathbf{c}^{*}=(2,3,4,5,6,7,8,9)^{\top}$. Table 3 shows the numerical results for the following various starting points:

$$
\text { (a) } \mathbf{c}^{0}=(1,1,1,1,1,1,1,1)^{\top} \text {, }
$$

(b) $\quad c^{0}=(2.5,2.5,2.5,2.5,2.5,2.5,2.5,2.5)^{\top}$,

$$
\text { (c) } \mathbf{c}^{0}=(1,2,3,4,5,6,7,8)^{\top} \text {, }
$$

(d) $\quad c^{0}=(2,4,6,8,10,12,14,16)^{\top}$,
TABLE 2: Numerical results for Example 2.

where

$$
\begin{gathered}
\mathbf{c}_{1}^{*}=(2,3,4,5,6,7,8,9)^{\top}, \\
\mathbf{c}_{2}^{*}=(-2.7065,-7.0690,8.4130,1.1038,-3.6637, \\
8.8346,5.5078,9.1273)^{\top}, \\
\mathbf{c}_{3}^{*}=(1.2877,2.4677,4.5379,-6.1037,1.7930, \\
1.1093,1.1024,5.3320)^{\top}
\end{gathered}
$$

are the three accumulation points of this problem.

We observe from Tables 1-3 that our algorithm is convergent for different starting points. We also see that our algorithm converges to a solution of the IEP, which is not necessarily equal to the original one. An interesting question is to consider the performance of the algorithm when the starting point $\mathbf{c}^{0}$ is chosen as random vector, which needs future study.

\section{Conflict of Interests}

The authors declare that there is no conflict of interests regarding the publication of this paper. 
TABLE 3: Numerical results for Example 3.

\begin{tabular}{|c|c|c|c|c|}
\hline$c^{0}$ & & $\beta=1.5$ & $\beta=1.8$ & $\beta=2.0$ \\
\hline \multirow{5}{*}{ (a) } & & $5.2594 e-03$ & $2.2949 e-04$ & $6.3777 e-02$ \\
\hline & errs & $1.5831 e-08$ & $2.1853 e-10$ & $5.2251 e-05$ \\
\hline & & $9.8210 e-12$ & $5.3338 e-12$ & $1.1737 e-11$ \\
\hline & ite. & 17 & 17 & 17 \\
\hline & $\mathrm{c}^{*}$ & $c_{2}^{*}$ & $\mathrm{c}_{3}^{*}$ & $\mathbf{c}_{2}^{*}$ \\
\hline \multirow{5}{*}{ (b) } & & $2.3747 e-02$ & $2.4283 e-02$ & $5.3326 e-02$ \\
\hline & errs & $6.6052 e-07$ & $6.8845 e-07$ & $3.5462 e-05$ \\
\hline & & $9.2871 e-12$ & $7.8077 e-12$ & $1.2096 e-11$ \\
\hline & ite. & 13 & 14 & 14 \\
\hline & $c^{*}$ & $\mathrm{c}_{2}^{*}$ & $\mathrm{c}_{2}^{*}$ & $\mathrm{c}_{2}^{*}$ \\
\hline \multirow{5}{*}{ (c) } & & $5.0900 e-04$ & $5.0902 e-04$ & $5.0902 e-04$ \\
\hline & errs & $5.5760 e-09$ & $5.5819 e-09$ & $5.5827 e-09$ \\
\hline & & $1.4600 e-11$ & $1.3617 e-11$ & $1.7533 e-11$ \\
\hline & ite. & 10 & 10 & 10 \\
\hline & $\mathrm{c}^{*}$ & $\mathbf{c}_{1}^{*}$ & $\mathrm{c}_{1}^{*}$ & $c_{1}^{*}$ \\
\hline \multirow{5}{*}{ (d) } & & $1.7014 e-02$ & $1.7014 e-02$ & $1.7014 e-02$ \\
\hline & errs & $6.9575 e-07$ & $6.9575 e-07$ & $6.9575 e-07$ \\
\hline & & $1.5224 e-11$ & $1.7256 e-11$ & $6.2077 e-11$ \\
\hline & ite. & 9 & 9 & 9 \\
\hline & $\mathrm{c}^{*}$ & $\mathrm{c}_{1}^{*}$ & $\mathrm{c}_{1}^{*}$ & $\mathrm{c}_{1}^{*}$ \\
\hline
\end{tabular}

\section{Acknowledgment}

The second author's work was supported in part by the National Natural Science Foundation of China (Grant no. 61170109).

\section{References}

[1] F. Diele, T. Laudadio, and N. Mastronardi, "On some inverse eigenvalue problems with Toeplitz-related structure," SIAM Journal on Matrix Analysis and Applications, vol. 26, no. 1, pp. 285-294, 2004.

[2] W. F. Trench, "Numerical solution of the inverse eigenvalue problem for real symmetric Toeplitz matrices," SIAM Journal on Scientific Computing, vol. 18, no. 6, pp. 1722-1736, 1997.

[3] M. T. Chu, "Inverse eigenvalue problems," SIAM Review, vol. 40, no. 1, pp. 1-39, 1998.

[4] M. T. Chu and G. H. Golub, "Structured inverse eigenvalue problems," Acta Numerica, vol. 11, pp. 1-71, 2002.

[5] M. T. Chu and G. H. Golub, Inverse Eigenvalue Problems: Theory, Algorithm and Application, Oxford University Press, New York, NY, USA, 2005.

[6] S. Friedland, J. Nocedal, and M. L. Overton, "The formulation and analysis of numerical methods for inverse eigenvalue problems," SIAM Journal on Numerical Analysis, vol. 24, no. 3, pp. 634-667, 1987.

[7] R. H. Chan, H. L. Chung, and S.-F. Xu, "The inexact Newtonlike method for inverse eigenvalue problem," BIT, vol. 43, no. 1, pp. 7-20, 2003.

[8] R. H. Chan, S.-F. Xu, and H.-M. Zhou, "On the convergence of a quasi-Newton method for inverse eigenvalue problems," SIAM Journal on Numerical Analysis, vol. 36, no. 2, pp. 436-441, 1999.
[9] Z.-J. Bai, R. H. Chan, and B. Morini, "An inexact Cayley transform method for inverse eigenvalue problems," Inverse Problems, vol. 20, no. 5, pp. 1675-1689, 2004.

[10] W. Shen and C. Li, "An Ulm-like Cayley transform method for inverse eigenvalue problems," Taiwanese Journal of Mathematics, vol. 16, no. 1, pp. 367-386, 2012.

[11] W. P. Shen, C. Li, and X. Q. Jin, "A Ulm-like method for inverse eigenvalue problems," Applied Numerical Mathematics, vol. 61, no. 3, pp. 356-367, 2011.

[12] S. C. Eisenstat and H. F. Walker, "Globally convergent inexact Newton methods," SIAM Journal on Optimization, vol. 4, no. 2, pp. 393-422, 1994.

[13] S. C. Eisenstat and H. F. Walker, "Choosing the forcing terms in an inexact Newton method," SIAM Journal on Scientific Computing, vol. 17, no. 1, pp. 16-32, 1996.

[14] M. Pernice and H. F. Walker, "NITSOL: a Newton iterative solver for nonlinear systems," SIAM Journal on Scientific Computing, vol. 19, no. 1, pp. 302-318, 1998.

[15] J. E. Dennis, Jr. and R. B. Schnabel, Numerical Methods for Unconstrained Optimization and Nonlinear Equations, Society for Industrial and Applied Mathematics (SIAM), Philadelphia, Pa, USA, 2nd edition, 1996.

[16] S. Bellavia and B. Morini, "A globally convergent NewtonGMRES subspace method for systems of nonlinear equations," SIAM Journal on Scientific Computing, vol. 23, no. 3, pp. 940960, 2001.

[17] Z.-J. Bai, B. Morini, and S.-F. Xu, "On the local convergence of an iterative approach for inverse singular value problems," Journal of Computational and Applied Mathematics, vol. 198, no. 2, pp. 344-360, 2007.

[18] J. G. Sun, "Eigenvalues and eigenvectors of a matrix dependent on several parameters," Journal of Computational Mathematics, vol. 3, no. 4, pp. 351-364, 1985.

[19] D. Sun and J. Sun, "Strong semismoothness of eigenvalues of symmetric matrices and its application to inverse eigenvalue problems," SIAM Journal on Numerical Analysis, vol. 40, no. 6 , pp. 2352-2367, 2003.

[20] R. W. Freund and N. M. Nachtigal, "QMR: a quasi-minimal residual method for non-Hermitian linear systems," Numerische Mathematik, vol. 60, no. 3, pp. 315-339, 1991. 


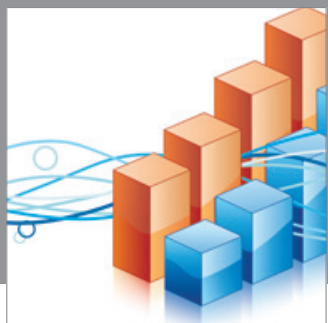

Advances in

Operations Research

mansans

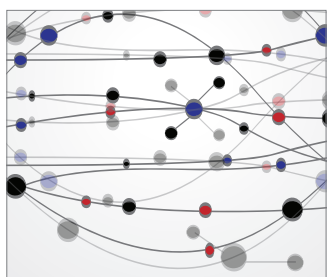

The Scientific World Journal
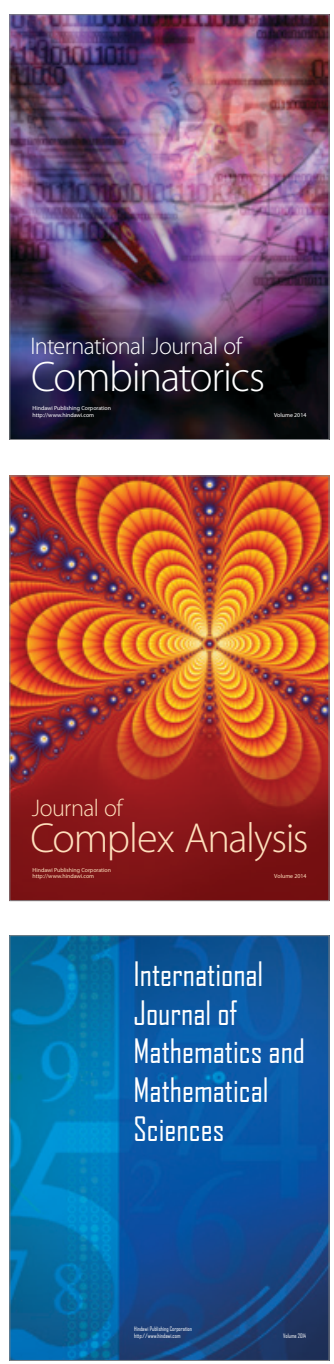
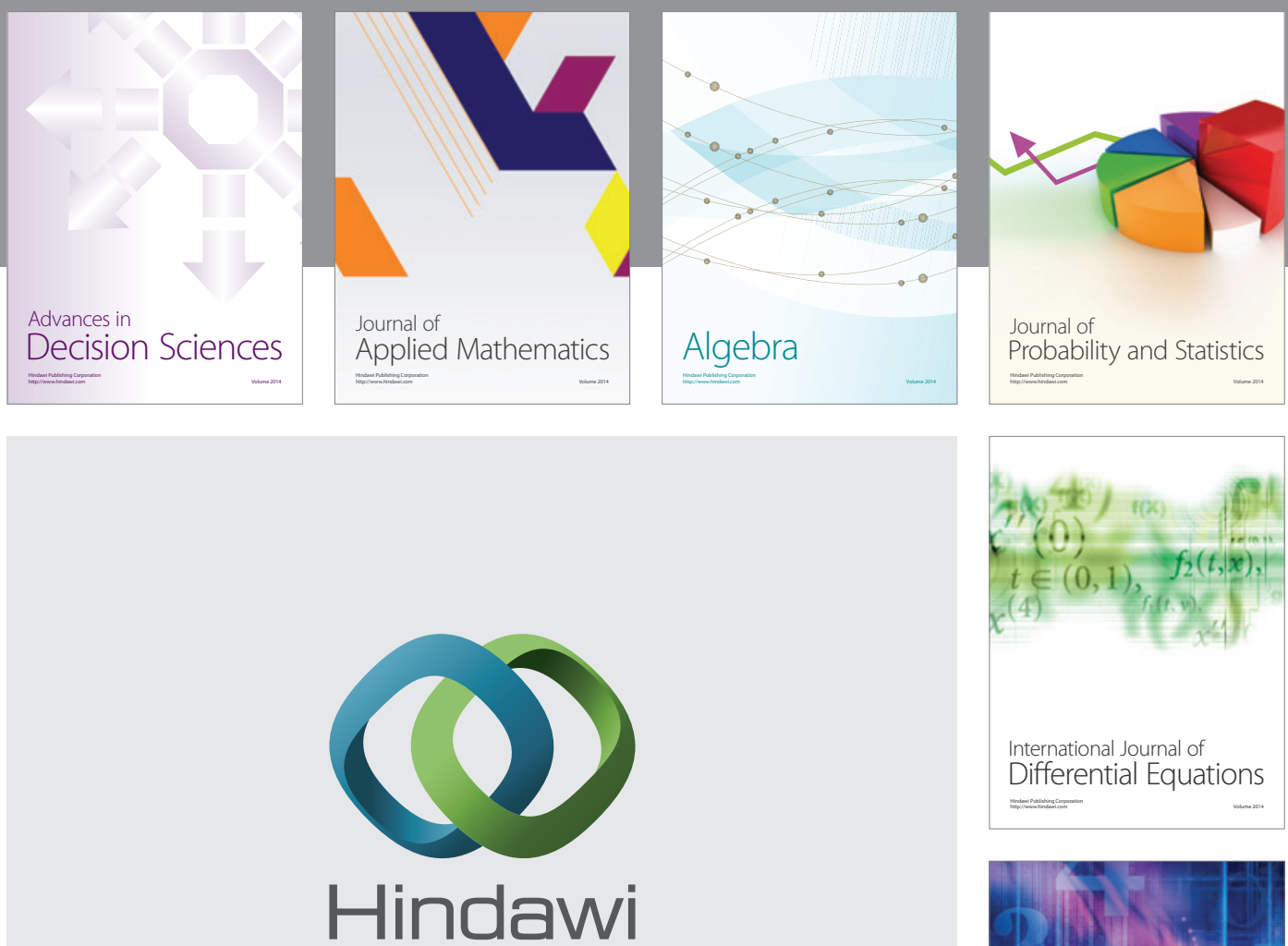

Submit your manuscripts at http://www.hindawi.com
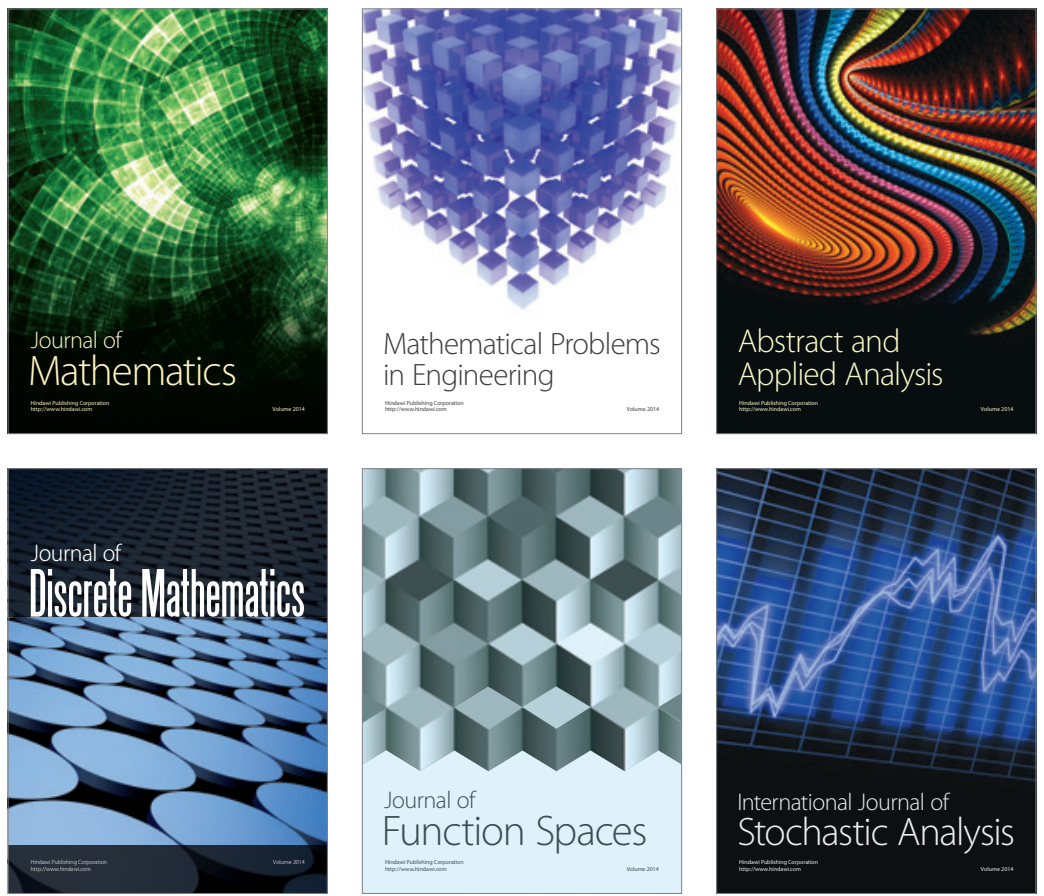

Journal of

Function Spaces

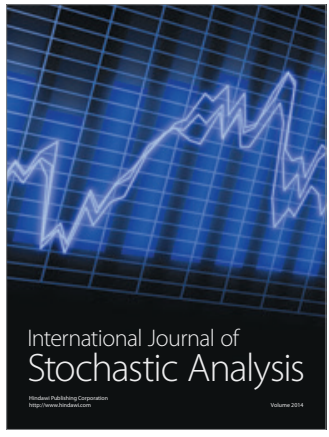

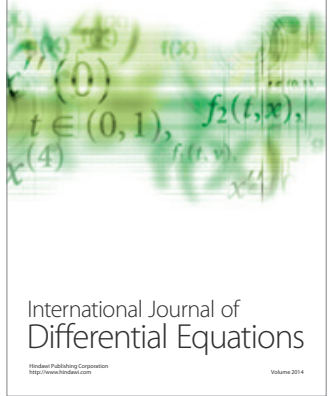
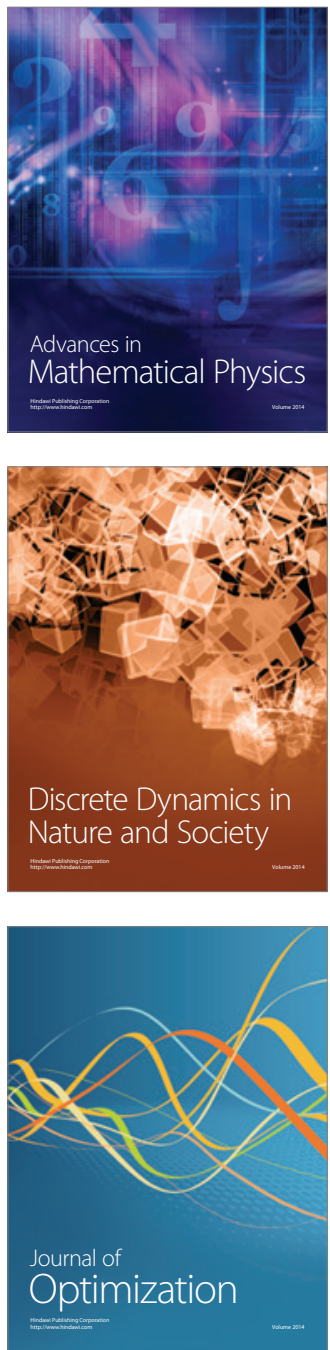